\title{
Entanglement Dynamics of Second Quantized Quantum Fields
}

\author{
Mikhail Erementchouk and Michael N. Leuenberger \\ NanoScience Technology Center, Department of Physics, University of Central Florida, Orlando, FL 32826, USA
}

Correspondence should be addressed to Mikhail Erementchouk; merement@gmail.com

Received 26 November 2013; Accepted 16 December 2013; Published 28 January 2014

Academic Editors: B. Bagchi, G. Berginc, and M. Sebawe Abdalla

Copyright (C) 2014 M. Erementchouk and M. N. Leuenberger. This is an open access article distributed under the Creative Commons Attribution License, which permits unrestricted use, distribution, and reproduction in any medium, provided the original work is properly cited.

\begin{abstract}
We study the entanglement dynamics in the system of coupled boson fields. We demonstrate that there are different natural notions of locality in this context leading to inequivalent notions of entanglement. We concentrate on the particle picture, when entanglement of one particle is determined by one-particle density matrix. We study, in detail, the effect of interaction preserving populations of individual one-particle states. We show that if the system is initially in a disentangled state with the definite total number of particles and the dimension of the one-particle Hilbert space is more than two, then only potentials of the special form admit complete entanglement, which is shown to be reached at NOON states. If the system is initially in Glauber's coherent state, complete entanglement is not reached despite the presence of two entangling channels in this case. We conclude with studying the time evolution of entanglement of photons in a cavity with multiple quantum dots in the limit of large number of photons. We show that in a relatively short time scale the completely entangled states belong to the class of graph states and are formed due to the interaction with dots in resonance with the cavity modes.
\end{abstract}

\section{Introduction}

Entanglement [1] is a quintessentially quantum feature. It signifies that different parts of a compound system may form a new entity, a complex; for example, when neither of two particles can be characterized by a definite state so that instead of two particles one has to consider a pair and so on. If one would attempt to access a particular part of the complex by performing a local measurement, this would unavoidably modify the state of other parts even if the direct interaction between the parts is absent or negligible. Such departure from the classical properties makes entanglement the central object in various contexts, from the perspective of application in quantum informatics [2] to understanding the physics of quantum phase transitions [3]. As a problem of special interest, therefore, the problem of preparation of a system in an entangled state stands out. Among different appearances of this problem, entangled states of quantized electromagnetic field, as perhaps the most accessible and the most flexible object, presents a significant importance on its own. Nowadays, the most developed and widely used method of generating entangled photons is the parametric down conversion [4-6], which is based on the two-photon radiative decay of material excited states. This method, however, suffers from well recognized intrinsic limitations such as very low yield and rescaling the wavelength of the emitted photons $[2,7,8]$. Therefore, there is the constant search for alternative sources of entangled light [9-19], which motivates a thorough consideration of entanglement of quantum many-body states.

The characteristic feature of the process of entangling photons in the course of interaction with matter is the nonconserving number of photons, or involved particles in general. In the few particle limit or in the case when the typical time scales are well separated, these processes can be reduced to a more or less standard quantum mechanical situation. This makes the process of entangling fit into the well developed description of entanglement. Indeed, in this case one can specify time periods when the system is either in the state of excited matter and no (relevant) photon, or in the state when matter is in the ground state and there are emitted photons. The general problem of solid based sources of entangled light, however, requires addressing the more general situation, when the photon states coexist with 
material excitations and the processes of reabsorption and reemission may play an important role. In this case one has to incorporate the nonconserving number of particles fully into consideration. The quantum field description (more precisely, the formalism of second quantization) provides the most natural framework for dealing with this kind of a situation. In this approach particles appear not as predefined entities as, say, qubits within the standard quantum mechanical treatment, but rather as excitations of the respective quantized fields.

As we will demonstrate below, entanglement in the context of quantized fields has its subtleties. The complete description of entanglement in this context is yet to be developed. For our purposes, however, a basic approach is sufficient. First, we will restrict our attention only to the case when the whole system can be characterized by a pure state. Second, entanglement will be considered from the perspective of the problem of sources of entangled light, which advances consideration of particle properties, as will be elaborated below. From this point of view the existence of entanglement implies that a set of particles is in a nonseparable state and, as a result, one particle cannot be described by a state vector; the particle of necessity is in a mixed state. Pure and mixed states, in turn, can be distinguished using the fact that extremal values of observables corresponding to operators with a nondegenerate spectrum are reached at pure states. Let $\widehat{O}$ be an operator acting on a finite dimensional Hilbert space and let its spectral decomposition be $\widehat{O}=$ $\sum_{\kappa} O_{\kappa} \widehat{\Pi}_{\kappa}$, where $O_{\kappa}$ are the eigenvalues, enumerated in the ascending order, $O_{1}<O_{2} \leq O_{3} \cdots \leq O_{N}$, and $\widehat{\Pi}_{\kappa}$ are the projectors on the respective eigenspaces. Furthermore, let the smallest eigenvalue be nondegenerate, that is, $\operatorname{rank}\left(\widehat{\Pi}_{1}\right)=$ 1 , then the minimal value of $\langle\widehat{O}\rangle \equiv \operatorname{Tr}[\widehat{\rho} \widehat{O}]$ (understood as a function of state described by the density matrix $\hat{\rho}$ ) is equal to $O_{1}$ and is reached at $\hat{\rho}$ corresponding to the pure state, $\widehat{\rho}=\widehat{\Pi}_{1}$. Respectively, the value of the observable $\langle\widehat{O}\rangle$ on mixed states will always satisfy inequality $\langle\widehat{O}\rangle_{\text {mix }}>O_{1}$. Indeed, on the basis, where $\widehat{O}$ is diagonal, one has $\operatorname{Tr}[\widehat{\rho} \widehat{O}]=$ $\sum_{\kappa} O_{\kappa} \rho_{\kappa, \kappa} \geq O_{1} \sum_{\kappa} \rho_{\kappa, \kappa}=O_{1}$ with the equality reached only when all $\rho_{\kappa, \kappa}$ but $\rho_{1,1}$ are zero. Thus, in a sense any operator with nondegenerate extremal eigenvalues allows distinguishing between pure and mixed states. Using this observation one can implement the ideology of witnessing entanglement, which was originally developed in the context of the full compound system $[1,20]$ and later was extended to one particle description [21].

This approach can be directly applied to the quantum field description. In this case, the role of witnesses is played by respective one-particle operators and following the standard line of arguments (see, e.g., [22]) one can characterize entanglement of one particle with the rest of the system using one particle correlation matrix (OPCM) $G_{\kappa, \lambda}=\left\langle a_{\kappa}^{\dagger} a_{\lambda}\right\rangle$, where $\kappa$ and $\lambda$ enumerate one-particle states and $a_{\kappa}^{\dagger}$ and $a_{\lambda}$ create and destroy a particle in the respective states. Throughout the paper we incorporate the time dependence into the Heisenber, representation of the operators $a_{\kappa}(t)=\exp (i \mathscr{H} t) a_{\kappa} \exp (-i \mathscr{H} t)$, where $\mathscr{H}$ is the
Hamiltonian describing the whole system. Thus, the time dependence of the OPCM is given by

$$
G_{\kappa, \lambda}(t)=\left\langle a_{\kappa}^{\dagger}(t) a_{\lambda}(t)\right\rangle
$$

where the average is taken with respect to the initial state $\langle\cdots\rangle=\langle\psi(t=0)|\cdots| \psi(t=0)\rangle$.

In order to illustrate the difficulty of producing entangled states let us consider a simple example of a boson field driven by an external source

$$
\mathscr{H}=\sum_{\kappa}\left(\epsilon_{\kappa} a_{\kappa}^{\dagger} a_{\kappa}+r_{\kappa}(t) a_{\kappa}^{\dagger}+r_{\kappa}^{*}(t) a_{\kappa}\right),
$$

where $r_{\kappa}(t)$ are $c$-numbers characterizing the external source.

Before applying rigorous methods let us note that this system may look confusing if approached with the help of often employed arguments based on interference of different paths connecting the initial and final states. Indeed, considering that there are different ways to fill some particular state, say, with only two particles, one might expect that these two particles will become entangled, which, of course, is an incorrect conclusion.

In virtue of the discussion above, the absence of entanglement would be manifested by rank one of the OPCM $[23,24]$, while the rank of the OPCM can be easily investigated. The solutions of the operator equations of motion have the form

$$
a_{\kappa}(t)=a_{\kappa}(0) \exp \left(-i \epsilon_{\kappa} t\right)+R_{\kappa}(t)
$$

where $R_{\kappa}(t)=\int_{0}^{t} d t^{\prime} \exp \left[-i \epsilon_{\kappa}\left(t-t^{\prime}\right)\right] r_{\mathcal{\kappa}}^{*}\left(t^{\prime}\right)$ are $c$-number functions. Substituting this representation into (1) we find

$$
\begin{aligned}
G_{\kappa, \lambda}(t)= & e^{i t\left(\epsilon_{\kappa}-\epsilon_{\lambda}\right)} G_{\kappa, \lambda}(0)+R_{\kappa}^{*}(t)\left\langle a_{\lambda}\right\rangle e^{-i \epsilon_{\lambda} t} \\
& +\left\langle a_{\kappa}^{\dagger}\right\rangle R_{\lambda}(t) e^{i \epsilon_{\kappa} t}+R_{\kappa, \lambda}(t)
\end{aligned}
$$

where $R_{\kappa, \lambda}(t)=R_{\kappa}^{*}(t) R_{\lambda}(t)$. Generally the structure of the OPCM $\widehat{G}(t)$ (throughout the paper hats denote matrices in one-particle Hilbert space) driven by the external source depends on time nontrivially. The last three terms in (4) may lead to variation of entanglement depending on the structure of the initial state. If, however, the system is initially in the vacuum state, that is, $\widehat{G}(0)=0$, then $\widehat{G}(t)=\widehat{R}(t)$, where $\widehat{R}(t)$ is the matrix with the elements $R_{\kappa, \lambda}(t)$. In turn, $\widehat{R}(t)$ is at most of rank which implies the absence of entanglement.

This example shows that the problem of the dynamics of entanglement should be treated with certain care. First, the naive arguments based on the picture of interference of different paths may be misleading. Second, no matter how complex the internal dynamics of the system described by the spectrum, $\epsilon_{\kappa}$, and independently on particular time dependence of the external excitation $r_{\kappa}(t)$, the states, reached out of vacuum under the action of this excitation, are disentangled. In particular, the initial state, which may lead to a nontrivial time dependence of entanglement, as has been mentioned above, must be created by means other than the external classical excitation.

The rest of the paper is organized as follows. In Section 2, we provide a more detailed description of entanglement in 
the context of quantized fields. In Section 3 we consider the entanglement dynamics for the system of linearly coupled boson fields. In Section 4 we study entanglement dynamics for the case of self-interacting boson field. Finally, in Section 5 we apply the obtained results to the analysis of the entanglement dynamics of photons in a cavity with multiple quantum dots in the limit of a large number of photons.

\section{Entanglement within Field and Particle Pictures}

Some results presented in Sections 3 and 4 may seem to contradict some results readily available in the literature. This reflects a certain ambiguity of the notion of entanglement in the context of quantized fields. Therefore, in order to avoid a possible misunderstanding, it is worthwhile to analyze the problem of entanglement in detail.

2.1. Field and Particle Pictures. The ambiguity stems from the fact that entanglement is understood as a relation between a part of the compound system and the whole system, while there are two distinctive notions of the part when quantized fields are considered: fields and particles. Which of these two different entities, fields and particles, appear more naturally is dictated by the physical content of the specific problem. For example, if a system of harmonic or anharmonic oscillators is considered [25], individual oscillators (i.e., fields) stand out as part of the big system, while, say, the dynamics of excitons in semiconductors $[10,12,14,16,17,19]$ promotes consideration of particles.

An arbitrary (pure) state of a system of $n$ (boson) fields can be specified in terms of degrees of excitation (population numbers) of each field

$$
|\psi\rangle=\sum_{N_{1}, \ldots, N_{n}} \Psi_{N_{1}, \ldots, N_{N}}\left|N_{1}, \ldots, N_{n}\right\rangle
$$

where $N_{\kappa}$ denotes the population of the $\kappa$-th field and $\Psi_{N_{1}, \ldots, N_{n}}$ are the respective amplitudes. Equivalently, this state can be presented in terms of the particle creation operators

$$
|\psi\rangle=\sum_{N=0}^{\infty} \sum_{\kappa_{1}, \ldots, \kappa_{N}} \Phi_{\kappa_{1}, \ldots, \kappa_{N}} a_{\kappa_{1}}^{\dagger} \cdots a_{\kappa_{N}}^{\dagger}|0\rangle
$$

where $|0\rangle$ is vacuum, $N$ is the total number of particles, and $a_{\kappa}^{\dagger}$ creates a particle in the $\kappa$-th one-particle state. Representations (5) and (6) are equivalent if the amplitudes $\Phi_{\kappa_{1}, \ldots, \kappa_{N}}$ satisfy conditions that follow from the commutation of the creation operators $\left[a_{\kappa}^{\dagger}, a_{\lambda}^{\dagger}\right]=0$ and the relation between the individual fields in (5) and one-particle states in (6), $\left|N_{\kappa}\right\rangle=\left(a_{\kappa}^{\dagger}\right)^{N_{\kappa}}|0\rangle / \sqrt{N_{\kappa} !}$

While representations (5) and (6) are equivalent they imply different notions of "locality." This follows simply from the following observation. An operator acting on a particular field in (5) does not preserve the total number of particles unless it is proportional to the identity operator. At the same time an operator, which changes the state of one particle in (6), obviously affects the population numbers of more than one field. In turn, entanglement strongly relies on the notion of locality and, therefore, is sensitive to the choice of eligible local transformations. Therefore, one should distinguish representations (5) and (6). For this reason we will call representation (5) the field picture and (6) will be referred to as the particle picture. Most of the papers dealing with entanglement in the field context are effectively restricted to one of the pictures implied by the physical situation. This, however, leads to possible ambiguities because results are often formulated in some general terms (entanglement, part, compound system, and so on), which are identical for both pictures. Entanglement, however, does depend on the picture, as is suggested by the nonequivalence of the notions of locality and will be demonstrated below.

Within the field picture, if the general form of operators acting on a particular field is allowed, entanglement is naturally related to separability of amplitudes $\Psi_{N_{1}, \ldots, N_{n}}$. In systems with superselection rules [26], the space of allowed operators is "smaller" and the operators, whose mean value is extremal at the particular state, may not be accessible. From the perspective of witness ideology, this means that such state should be considered as entangled. For such systems, therefore, one needs a criteria of entanglement different from the mere separability of the respective amplitudes. This problem, however, goes beyond the scope of the present paper.

In order to quantify entanglement of the $\kappa$-th field with the rest of the system, that is, with the other fields, it is convenient to introduce the reduced density matrix

$$
\begin{aligned}
\rho_{N, N^{\prime}}^{(\kappa)}=\sum_{N_{1}, \ldots, N_{n}}\langle & N_{1}, \ldots, N_{\kappa-1}, N, \ldots, N_{n}|\psi\rangle \\
& \times\left\langle\psi \mid N_{1}, \ldots, N_{\kappa-1}, N^{\prime}, \ldots, N_{n}\right\rangle,
\end{aligned}
$$

where the summation excludes $N_{\kappa}$. If the rank of the reduced density matrix $\rho_{N, N^{\prime}}^{(\kappa)}$ is higher than one, we have entanglement, which can be quantified, for example, by the von Neumann entropy. Thus, entanglement in the field picture fits the canonical quantum mechanical description based on separability of the amplitudes and agrees with the general witness ideology.

Within the particle picture, however, one has to rely upon the witness approach more due to indistinguishability of individual particles, which makes the separability property of amplitudes $\Phi_{\kappa_{1}, \ldots, \kappa_{N}}$ an inadequate criterium. The quantity of main interest becomes the one-particle correlation matrix

$$
G_{\kappa, \lambda}=\left\langle a_{\kappa}^{\dagger} a_{\lambda}\right\rangle
$$

which is related to the amplitudes $\Phi_{\kappa_{1}, \ldots, \kappa_{N}}$ in a rather complex way. The one-particle density matrix (OPDM) is defined as a properly normalized correlation matrix $\hat{\rho}=$ $\widehat{G} / \operatorname{Tr}[\widehat{G}]$. Then, entanglement can be quantified, for example, by von Neumann entropy (we will return to the question of the entanglement measure below) $E_{N}[\hat{\rho}]=-\operatorname{Tr}[\hat{\rho} \ln (\hat{\rho})]$. One can easily check that when the system immediately admits the standard description (e.g. when all particles are distinguishable, i.e., all $\kappa_{i}$ in (6) are different, and the field 
and particle pictures are identical) the approach based on the OPDM yields results consistent with this description. Of course, OPDM may only answer questions regarding entanglement of a single particle with the rest of the system. If one is interested in more subtle details, such as, for instance, entanglement in pairs, one has to look at the density matrices of higher orders. We, however, limit ourselves to studying the basic properties of entanglement and for this purpose it suffices to consider OPDM, which can be shown to yield the upper bound for entanglement in the system.

The necessity to distinguish entanglement within different pictures is illustrated by states with amplitudes (in the field picture) being separable, $\Psi_{N_{1}, \ldots, N_{n}}=\prod_{\kappa=1}^{n} \psi_{N_{\kappa}}^{(\kappa)}$, and for each field only amplitudes of the same parity are not zero, for example, $\psi_{2 k}^{(\kappa)}=0$ for all $\kappa$ and integral $k \geq 0$. These states are disentangled within the field picture; they, however, may be completely entangled within the particle picture. Indeed, the off-diagonal elements of the OPDM vanish for such states and if, additionally, the amplitudes $\psi_{2 k+1}^{(\kappa)}$ are chosen in such manner that the average number of particles in each state (the degree of excitation of each field) is the same, $\left\langle a_{\kappa}^{\dagger} a_{\kappa}\right\rangle=$ const, then the von Neumann entropy takes the maximal value, implying maximal entanglement within the particle picture.

The example of opposite situations is presented by singleparticle entanglement [21, 27-30], when, say, $\Psi_{1,0} \Psi_{0,1} \neq 0$, while all other amplitudes in (5) are zero. These states are obviously entangled within the field picture while disentangled in the framework of the particle picture.

\subsection{Disentangled States in Particle Picture. For a more de-} tailed comparison of different pictures we show that the states disentangled within both field and particle pictures constitute the special class and this class is not particularly rich. Besides the states with only excitations of one field, when trivially there is nothing to entangle (we would like to remind the reader that we consider the case without superselection rules), are canonical coherent states [31-33].

First of all we describe all disentangled states within the particle picture. The main result here is almost obvious: particles are disentangled within the particle picture if and only if they all are in the same state. In order to prove this statement (nontrivial in the part that there are no other disentangled states) it is convenient to introduce special notations.

We introduce a vector-operator a with components $(\mathbf{a})_{\mathcal{\kappa}}=$ $a_{\kappa}$. The commutation relation in terms of the vector-operators can be formally written as $\left[\mathbf{a}^{\dagger}, \mathbf{a}\right]=-\hat{1}$. These are vectors in the following sense. The choice of a different set of basis one-particle states in space $H$ corresponds to a linear transformation of $H$, which translates into choosing a different set of operators $b_{\lambda}$ linearly related to the old set

$$
b_{\lambda}=\sum_{\kappa} a_{\kappa} U_{\kappa, \lambda}
$$

or $\mathbf{b}=\mathbf{a} \widehat{U}$. The commutation relation for new operators can be shown to have the form $\left[\mathbf{b}^{\dagger}, \mathbf{b}\right]=-\widehat{U}^{\dagger} \widehat{U}$. Thus, in order to satisfy the boson commutation relation, the vector-operators b and a must be related through a unitary transformation.
These transformations, in particular, preserve the operator of the total number of particles. This can be illustrated by presenting the operator as $\mathcal{N}=\sum_{\kappa} a_{\kappa}^{\dagger} a_{\kappa} \equiv \operatorname{Tr}\left(\mathbf{a}^{\dagger} \otimes \mathbf{a}\right)$. In this section $\otimes$ denotes the product $\left(\mathbf{a}^{\dagger} \otimes \mathbf{a}\right)_{\kappa, \lambda}=a_{\kappa}^{\dagger} a_{\lambda}$, which transforms as a tensor.

The reason why we have introduced vector-operators is that OPCM also transforms as a tensor

$$
\left\langle\mathbf{b}^{\dagger} \otimes \mathbf{b}\right\rangle=\widehat{U}^{\dagger}\left\langle\mathbf{a}^{\dagger} \otimes \mathbf{a}\right\rangle \widehat{U}
$$

that is, $\widehat{G}$ is mapped into $\widehat{U}^{\dagger} \widehat{G U}$. Thus, unitary transformations do not change the spectrum of OPCM and, due to invariance of $\mathcal{N}$, they leave entanglement intact.

The important consequence of this geometrical picture is that it immediately provides the description of all disentangled states. OPCM is a Hermitian matrix and, therefore, can be diagonalized by a unitary transformation, implying the existence of the preferred set of operators. In turn, OPCM of any disentangled state in the diagonal form has only single nonzero element. Thus, choosing the appropriate transformation we can have only $\left\langle b_{1}^{\dagger} b_{1}\right\rangle \neq 0$ while all other elements of OPCM are zero. The only states yielding such OPCM are of the form

$$
|\psi\rangle=\sum_{n} \frac{\phi_{n}}{\sqrt{n !}}\left(b_{1}^{\dagger}\right)^{n}|0\rangle
$$

where $\sum_{n}\left|\phi_{n}\right|^{2}=1$. Using relation (9) we can expand $b_{1}^{\dagger}=$ $\sum_{\kappa} U_{\kappa, 1}^{*} a_{\kappa}^{\dagger}$. As follows from unitarity, the single column of a unitary matrix is a unit vector. Denoting this vector by $\mathbf{S}$ and defining the "scalar" product $\mathbf{S} \cdot \mathbf{a}^{\dagger} \equiv \sum_{\kappa} S_{\kappa} a_{\kappa}^{\dagger}$, we can parametrize all disentangled states

$$
\left|\psi\left(\mathbf{S} ; \phi_{1}, \ldots\right)\right\rangle=\sum_{N} \frac{\phi_{N}}{\sqrt{N !}}\left(\mathbf{S} \cdot \mathbf{a}^{\dagger}\right)^{N}|0\rangle
$$

by a set of amplitudes $\phi_{N}$ and a vector on the unit sphere in $\mathbb{C}^{n}$. There is a one-to-one correspondence between such sphere and the space of one-particle states; therefore, we will sometimes refer to vector $\mathbf{S}$ as a state. Disentangled states with a definite number of particles (with $\phi_{N}=0$ for all but specific $N)$ will play an important role in the following consideration. Extending the terminology used for the case $n=2$ we will call them spin coherent states.

Once we have established the general form of disentangled states in the particle picture we may proceed and find which of those are disentangled in the field picture. In order to find amplitudes $\Phi_{N_{1}, \ldots, N_{n}}=\left\langle N_{1}, \ldots, N_{M} \mid \psi(\mathbf{S})\right\rangle$ we first notice that only the term with the same total number of particles $N=\sum_{i} N_{i}$ in (12) contributes to $\Phi_{N_{1}, \ldots, N_{n}}$ and from the polynomial expansion of this term we need only one term with matching population numbers for each field. Thus, we find

$$
\Phi_{N_{1}, \ldots, N_{n}}=\phi_{N} \sqrt{N !} \prod_{\kappa} S_{\kappa}^{N_{\kappa}} \sqrt{N_{\kappa} !}
$$

with $N=\sum_{\kappa} N_{\kappa}$ and the important convention that $S_{\kappa}^{0}=$ 1 even if $S_{\kappa}=0$. It follows from (13) that there are only 
two possibilities to have separability of $\Phi_{N_{1}, \ldots, N_{n}}$. The first one is when all but one $S_{\kappa}$ are zero. This corresponds to the trivial case when only the single type of fields is excited. The second possibility is when $\phi_{N}=c \alpha^{N} / \sqrt{N !}$ with some complex numbers $c$ and $\alpha$. Substituting these amplitudes into (12) and enforcing the normalization condition we find that all states disentangled in both field and particle pictures can be presented as

$$
|\psi(\mathbf{S} ; \alpha)\rangle=\exp \left(\alpha \mathbf{S} \cdot \mathbf{a}^{\dagger}-\alpha^{*} \mathbf{S}^{*} \cdot \mathbf{a}\right)|0\rangle
$$

These are Glauber's coherent states. It is interesting to emphasize a relation with the example considered in the introduction. Hamiltonian (2) is diagonalized by introducing operators $b_{\kappa}=a_{\kappa}+r_{\kappa} / \epsilon_{\kappa}$, which are obtained by employing Glauber's shift operator $\exp \left(\alpha \mathbf{S} \cdot \mathbf{a}^{\dagger}-\alpha^{*} \mathbf{S}^{*} \cdot \mathbf{a}\right)$ with $\alpha S_{\kappa}=$ $r_{\kappa} / \epsilon_{\kappa}$. This, in particular, proves that states reached from vacuum under classical excitation are disentangled within both pictures.

2.3. NOON States. The general "inverse" problem of a relation between entanglement and the structure of respective states requires consideration, which goes far beyond the objectives of the present paper. Therefore we limit ourselves to an explicit description of a few sets of completely entangled states.

In Section 4 states of NOON type appear. While these states were introduced for qubits [34], they can be defined in a more general setup as follows. Let vectors $\mathbf{S}(\kappa), \kappa=1, \ldots, n$ form a basis in $\mathbb{C}^{n}$. Then NOON states of $N$ particles with $n$ dimensional one-particle Hilbert space are defined as

$$
|\mathrm{NOON}\rangle=\frac{1}{\sqrt{n N !}} \sum_{\kappa}\left[\mathbf{S}(\kappa) \cdot \mathbf{a}^{\dagger}\right]^{N}|0\rangle
$$

Thus, taking into account that different rearrangements of $\mathbf{S}(\kappa)$ produce the same state, the manifold of NOON states is isomorphic to $S U(n) / S_{n}$, where $S_{n}$ is the symmetric group (group of all permutations of $n$ elements).

It should be noted that NOON states do not exhaust all completely entangled states. In order to see this it is useful to present NOON states as they appear in a more general context. The natural representation of $S_{n}$ on the basis $\mathbf{S}(\kappa)$ has the form $T(g) \mathbf{S}(\kappa)=\mathbf{S}\left(g^{-1} \kappa\right)$ for any $g \in S_{n}$. Taking any $1 \leq \kappa \leq M$, NOON states can be defined in terms of the orbits

$$
|\mathrm{NOON}\rangle=\frac{\sqrt{n}}{n ! \sqrt{N !}} \sum_{g \in S_{n}}\left[\mathbf{S}\left(g^{-1} \kappa\right) \cdot \mathbf{a}^{\dagger}\right]^{N}|0\rangle
$$

where we have taken into account that the orbit $g^{-1} \kappa$ on $\{1, \ldots, n\}$ visits each element $(n-1)$ ! times as $g$ runs over $S_{n}$. Representation (16) demonstrates that NOON states are a particular class of entangled coherent states $[35,36]$. Namely, NOON states are equally weighted superpositions of orthogonal spin coherent states.
Using the same approach as for (16) another set of completely entangled states can be constructed for $N>3$. For any pair $1 \leq \kappa, \lambda \leq n$ such that $\lambda \neq \kappa$ states

$$
\begin{aligned}
|N-1,1\rangle= & \frac{\sqrt{n}}{n ! \sqrt{(N-1) !}} \\
& \times \sum_{g \in S_{n}}\left[\mathbf{S}\left(g^{-1} \kappa\right) \cdot \mathbf{a}^{\dagger}\right]^{N-1} \mathbf{S}\left(g^{-1} \lambda\right) \cdot \mathbf{a}^{\dagger}|0\rangle
\end{aligned}
$$

are completely entangled. This makes an interesting connection between completely entangled states (constituting a straightforward generalization of Dicke states $[37,38])$ and irreducible representations of symmetric group $S_{n}$.

2.4. One-Particle Entanglement from Lie-Algebraic Perspective. The natural framework for dealing with entanglement in particle picture is provided by the language of irreducible representations of $\mathfrak{s} \mathfrak{t}(n)$ Lie algebras. For example, in the case of a two-dimensional one-particle Hilbert space (e.g., photons characterized by two "+" and “-" polarizations), it is convenient to employ Schwinger's model of angular momentum [39] and to introduce

$$
\begin{aligned}
& \mathscr{J}_{x}=\frac{1}{2}\left(a_{+}^{\dagger} a_{-}+a_{-}^{\dagger} a_{+}\right), \\
& \mathscr{J}_{y}=\frac{1}{2 i}\left(a_{+}^{\dagger} a_{-}-a_{-}^{\dagger} a_{+}\right), \\
& \mathscr{J}_{z}=\frac{1}{2}\left(a_{+}^{\dagger} a_{+}-a_{-}^{\dagger} a_{-}\right),
\end{aligned}
$$

which satisfy the commutation relation of $\mathfrak{g} \mathfrak{t}(2)$ algebra $\left[\mathscr{F}_{\kappa}, \mathscr{J}_{\lambda}\right]=i \epsilon_{\kappa, \lambda, \mu} \mathscr{J}_{\mu}$ with $\epsilon_{\kappa, \lambda, \mu}$ being a completely antisymmetric tensor. These operators provide a representation of OPDM. Consider

$$
\widehat{\rho}=\frac{1}{2} \widehat{1}+\sum_{i} \sigma_{i}\left\langle\mathscr{J}_{i}\right\rangle,
$$

where $i$ runs over $\{x, y, z\}$ and $\sigma_{i}$ are Pauli matrices and $\sigma_{0}$ is the identity matrix. The sum over $\sigma_{i}$ describes a deviation of $\hat{\rho}$ from the identity matrix and hence a deviation of the state from a completely entangled one. This implies that entanglement can be expressed in terms of $J^{2}=\sum_{i}\left\langle\mathscr{J}_{i}\right\rangle^{2}$.

Before formulating a general approach let us consider a two-particle case, which provides a clear connection with the standard quantum-mechanical consideration. On the basis of the population numbers, any two-particle state can be presented as

$$
|\psi\rangle=\Psi_{2,0}|2,0\rangle+\Psi_{1,1}|1,1\rangle+\Psi_{0,2}|0,2\rangle,
$$

where $\left|N_{+}, N_{-}\right\rangle$denotes the state with $N_{+}$particles in the "+"mode and $N_{-}$particles in the "-"-mode. Alternatively, the state can be presented as [23]

$$
|\psi\rangle=\sum_{\kappa, \lambda} w_{\kappa, \lambda} a_{\kappa}^{\dagger} a_{\lambda}^{\dagger}|0\rangle
$$


where $w_{\kappa, \lambda}$ is a symmetric matrix. Comparing (20) and (21) one finds $w_{++}=\Psi_{2,0} / \sqrt{2}, w_{--}=\Psi_{0,2} / \sqrt{2}$ and $w_{+-}=\Psi_{1,1} / 2$. From (21) one obtains $\widehat{G}=4 \widehat{w}^{\dagger} \widehat{w}$.

The OPCM is a $2 \times 2$ matrix and, therefore, its eigenvalues are completely determined by $\operatorname{det}[\widehat{G}]=\lambda_{1} \lambda_{2}$ and $\operatorname{Tr}[\widehat{G}]=$ $\lambda_{1}+\lambda_{2}=N$ with $N$ being the total number of particles. Entanglement, in turn, is determined by the normalized eigenvalues $\tilde{\lambda}_{1,2}=\lambda_{1,2} / N$, which are found as

$$
\tilde{\lambda}_{1,2}=\frac{1}{2} \pm \frac{1}{2} \sqrt{1-\left|\frac{2 C}{N}\right|^{2}}
$$

where $|C|^{2}=\operatorname{det} \widehat{G}$ is the concurrence [40]. In order to see the relation with the standard definition of the concurrence in the two-particle case we introduce the "spin flip" transformation $\sigma_{y}|+\rangle=-i|-\rangle, \sigma_{y}|-\rangle=i|+\rangle$ and the spin flip state $|\widetilde{\psi}\rangle=$ $\left(\sigma_{y} \otimes \sigma_{y}|\psi\rangle\right)^{*}$, then $C=\langle\widetilde{\psi} \mid \psi\rangle=4 \operatorname{det}[\widehat{w}]$. Thus, the two-particle case completely fits into the canonical quantummechanical description and, moreover, concurrence is tightly connected to average angular momentum; for an arbitrary $\mathrm{N}$ one has $|C|^{2}=N^{2} / 4-J^{2}$.

Now we can turn to a general $S U(n)$ case. Let $T^{(i)}$ with $i=1, \ldots, n^{2}-1$ be generators of $\mathfrak{g} \mathfrak{t}(n)$, which are presented in $\mathbb{C}^{n}$ by traceless Hermitian matrices $\widehat{T}^{(i)}$ normalized according to $\operatorname{Tr}\left[\widehat{T}^{(i)} \widehat{T}^{(j)}\right]=\delta_{i, j} / 2[41]$. It can be checked that operators

$$
\mathscr{T}^{(i)}=\sum_{\kappa, \lambda} \widehat{T}_{\kappa, \lambda}^{(i)} a_{\kappa}^{\dagger} a_{\lambda}
$$

constitute a symmetric representation of $\mathfrak{g} \mathfrak{u}(n)$ on the boson Fock space and this representation is irreducible within each sector with a definite total number of particles.

Any Hermitian $n \times n$ matrix can be uniquely presented as a linear combination of $\widehat{T}^{(i)}$ and the identity matrix $\hat{1}$. Taking into account the chosen normalization of $\widehat{T}^{(i)}$ we find a representation of OPDM generalizing (19) [42]

$$
\widehat{\rho}=\frac{1}{n} \widehat{1}+\widetilde{\mathbf{J}} \cdot \widehat{\mathbf{T}},
$$

where $(\widehat{\mathbf{T}})_{i}=\widehat{T}^{(i)}$ and $\widetilde{\mathbf{J}}$ is a Bloch vector with components $\widetilde{J}_{i}=2\left\langle\mathscr{T}^{(i)}\right\rangle /\langle N\rangle$.

As follows from (24) vector $\widetilde{\mathbf{J}}$ completely determines the spectrum of OPDM and, therefore, any measure of entanglement in the particle picture is a function of $\widetilde{\mathbf{J}}$ or, more precisely, of its invariants with respect to adjoint representation of $S U(n)$ [42]. It is worth noting that since operators $\mathscr{T}^{(i)}$ correspond to a set of single-particle observables, this implies that entanglement is an observable.

Entanglement is a global characteristic of the spectrum of OPDM. Qualitatively, it is related to the density of the distribution of eigenvalues of $\hat{\rho}$; if they, including zeros, are far away from each other, entanglement is weak; if they are close, entanglement is strong. Taking into account that $\operatorname{Tr}[\hat{\rho}]=1$ the simplest characteristic of the spectrum is the averaged squared distance between the eigenvalues

$$
S(\widehat{\rho})=\frac{1}{2 n} \sum_{\kappa, \lambda}\left(\rho_{\kappa}-\rho_{\lambda}\right)^{2} .
$$

It changes from 0 , when all $\rho_{\kappa}=1 / n$ (complete entanglement), to $(n-1) / n$, when one eigenvalue is 1 and the remaining are 0 (absence of entanglement). By expanding (25) we find

$$
S(\widehat{\rho})=\sum_{\kappa} \rho_{\kappa}^{2}-\frac{1}{n}=\operatorname{Tr}\left[\hat{\rho}^{2}\right]-\frac{1}{n} .
$$

This establishes the relation between $S(\widehat{\rho})$ and the linear entropy

$$
E_{L}(\hat{\rho})=\frac{n}{n-1}\left(1-\operatorname{Tr}\left[\hat{\rho}^{2}\right]\right)=1-\frac{n S(\widehat{\rho})}{n-1} .
$$

On the other hand, from (24) we find $S(\widehat{\rho})=\widetilde{J}^{2} / 2$. Thus, $\widetilde{J}^{2}$ changes from 0 for completely entangled states to $2(n-1) / n$ for disentangled ones. The condition $\left\langle\mathscr{T}^{(i)}\right\rangle=0$ has been considered as a criterion for complete entanglement from different perspectives: maximization of quantum fluctuations [43] and geometric invariant theory [44]. We would like, therefore, to emphasize that within the present approach this condition is a consequence of the physical requirement that an entangled particle cannot be characterized by any definite one-particle state, which allows for a straightforward generalization for the case of indefinite number of particles.

Other measures of entanglement may involve more subtle characteristics of the distribution of the spectrum of OPDM and do not have to coincide with the linear entropy [45]. It should be noted, however, that the choice of essentially different measures is limited. Clearly, any measure should be invariant with respect to basis transformations and, therefore, should be a function of invariants of OPDM only. For $n \times$ $n$ matrices there are $n-1$ invariants determined by the exact positions of eigenvalues of OPDM constrained by the requirement $\operatorname{Tr}[\hat{\rho}]=1$. Thus, any measure can be expressed in terms of $\operatorname{Tr}\left[\hat{\rho}^{k}\right]$ with $k=2, \ldots, n-1$. In particular, for $S U(2)$ there is only one invariant and, therefore, any measure of entanglement is a function of a single parameter $\widetilde{J}$, which in this case has the meaning of the magnitude of average angular momentum. For example, in the $S U(2)$ case, entanglement without any loss of generality can be quantified by the linear entropy

$$
E_{L}=1-\widetilde{J}
$$

Moreover, if one is interested mostly in limiting situations of complete entanglement and disentanglement, a description employing only $\widetilde{J}$ is sufficient also for the case of general $S U(n)$. Therefore, in the following consideration, we will use the linear entropy for characterization of entanglement. It is especially convenient because $\operatorname{Tr}\left[\hat{\rho}^{2}\right]=\sum_{\kappa, \lambda}\left|\rho_{\kappa, \lambda}\right|^{2}$, which effectively allows one to calculate $\widetilde{J}$ without dealing with the problem of specific representations of $\mathfrak{\mathfrak { H }}(n)[46,47]$. 


\section{Entanglement Transfer between Coupled Fields}

We begin our analysis of dynamics of entanglement by considering a simple but important case of linearly coupled fields described by the Hamiltonian

$$
\begin{aligned}
\mathscr{H}= & \sum_{k} \epsilon_{k}^{(a)} a_{k}^{\dagger} a_{k}+\sum_{\kappa} \epsilon_{\kappa}^{(b)} b_{\kappa}^{\dagger} b_{\kappa} \\
& +\sum_{k, \kappa}\left(d_{k, \kappa} a_{k}^{\dagger} b_{\kappa}+d_{k, \kappa}^{*} b_{\kappa}^{\dagger} a_{k}\right),
\end{aligned}
$$

where $1 \leq k \leq n^{(a)}$ and $1 \leq \kappa \leq n^{(b)}$ enumerate the modes of the fields; $\epsilon_{k}^{(a)}, \epsilon_{\kappa}^{(b)}$, and $d_{k, \kappa}$ are the spectra of the fields and the coupling constants between them, respectively, and the operators $a_{k}$ and $b_{\kappa}$ are assumed to obey the boson commutation relations.

The important feature of the time evolution of entanglement in this system is that the total entanglement remains constant and is solely determined by the initial state. For a more precise formulation, instead of the operators $a_{k}$ and $b_{\kappa}$, let us introduce the combined operators $u_{i}$. That is instead of two fields with two sets of modes $M_{a}$ and $M_{b}$ we consider the single field, whose modes are the direct sum $M_{a} \oplus M_{b}$. Thus, $u_{i} \equiv a_{k_{i}}$ if $i \in M_{a}$ and $u_{i} \equiv b_{\kappa_{i}}$ if $i \in M_{b}$. For field $u$ we define the OPCM as

$$
G_{i j}(t)=\left\langle u_{i}^{\dagger}(t) u_{j}(t)\right\rangle .
$$

When both $i$ and $j$ belong to, say, $M_{a}$ the respective matrix elements $G_{i j}(t)$ give the OPCM for field $a$ and so on. Furthermore, let $\widehat{G}$ have the spectral representation

$$
\widehat{G}(t)=\sum_{l=1}^{n} \lambda_{l}(t) \mathbf{v}_{l}(t) \otimes \mathbf{v}_{l}(t)
$$

where $n=n^{(a)}+n^{(b)}$ and $\lambda_{l}$ and $\mathbf{v}_{l}$ are the eigenvalues and the unit eigenvectors and $\otimes$ denotes the tensor product, which is defined as $\left(\mathbf{v} \otimes \mathbf{v}^{\prime}\right)_{i j}=v_{i}^{*} v_{j}^{\prime}$. Then the total entanglement can be characterized by $E_{L}(t)=\left(1-\sum_{l} \widetilde{\lambda}_{l}^{2}\right) n /(n-1)$, where $\tilde{\lambda}_{l}(t)=\lambda_{l}(t) / \sum_{m} \lambda_{m}(t)$.

The important feature of systems with linear coupling between fields is that the entanglement evolution is restricted purely to its redistribution between the fields, while the total entanglement remains constant, $E_{L}(t)=E_{L}(0)$. This result while specific for the particle picture (thus contrasting the results obtained, e.g., in $[25,48])$ is in a more general context agreeable with the description of entanglement in terms of irreducible representations of $S U(n)$ Lie groups. Therefore, we prove it for a system described by the Hamiltonian $\mathscr{H}=\sum_{i j} h_{i j} u_{i}^{\dagger} u_{j}$, with Hermitian $\widehat{h}$ and pairs of field operators obeying the commutation relation $\left[u_{i}^{\dagger} u_{j}, u_{k}^{\dagger} u_{l}\right]=$ $u_{i}^{\dagger} u_{l} \delta_{k j}-u_{k}^{\dagger} u_{j} \delta_{i l}$. The total entanglement is determined by the spectrum of $\widehat{G}$, which satisfies $(\partial / \partial t) \widehat{G}(t)=i[\widehat{h}, \widehat{G}]$. The spectrum of matrices, whose time dependence is governed by such equations with the commutator in the r.h.s., does not change with time. Indeed, the solution has the form $\widehat{G}(t)=$ $\exp (\hat{i h} t) \widehat{G}(0) \exp (-i \widehat{h} t)$. Thus, due to unitarity of $\exp (i \widehat{h} t)$, the spectral representation of $\widehat{G}(t)$ is given by (31), with constant $\lambda_{l}$, and only the eigenvectors are functions of time. This implies that entanglement is an integral of motion.

For example, if initially there was only a single nonzero eigenvalue (i.e., entanglement was zero) it remains the only one later on, implying no production of entanglement. The same result holds for the OPCM corresponding to either fields $a$ or $b$. Indeed, the OPCM of field $a$ is obtained from the OPCM of the combined field $u$ applying the respective projection operators, $\widehat{\Pi}_{a}$, so that

$$
\widehat{G}^{(a)}=\widehat{\Pi}_{a} \widehat{G \Pi}_{a} \text {. }
$$

Using for $\widehat{G}$ its spectral representation one can see that such projection cannot increase the rank of the OPCM.

While inability of linear coupling to entangle initially disentangled fields can be expected [49], it should be noted that the evolution of entanglement of initially entangled states is nontrivial. As an example, let us consider the situation of small total entanglement, more specifically, when there are only two nonzero terms in (31) corresponding to $l=1,2$ with $\lambda_{1} \gg \lambda_{2}$. According to (32) the OPCM for field $a$ is given by

$$
\widehat{G}^{(a)}=\lambda_{1}^{(a)}(t) \mathbf{v}_{1}^{(a)}(t) \otimes \mathbf{v}_{1}^{(a)}(t)+\lambda_{2}^{(a)}(t) \mathbf{v}_{2}^{(a)}(t) \otimes \mathbf{v}_{2}^{(a)}(t),
$$

where $\mathbf{v}_{l}^{(a)}=\widehat{\Pi}_{a} \mathbf{v}_{l} /\left|\widehat{\Pi}_{a} \mathbf{v}_{l}\right|$ and $\lambda_{l}^{(a)}(t)=\lambda_{l}\left|\widehat{\Pi}_{a} \mathbf{v}_{l}\right|^{2}$. Thus, the value of entanglement depends on the magnitude of the projections $\widehat{\Pi}_{a} \mathbf{v}_{l}$, which is determined by the internal dynamics of the coupled fields. In particular, if $\left|\widehat{\Pi}_{a} \mathbf{v}_{l}\right| \ll 1$ one may have $\lambda_{1}^{(a)}(t) \sim \lambda_{2}^{(a)}(t)$, resulting in the significant entanglement of particles $a$, either with each other or with particles $b$. For more concrete information one has to take into account that, generally speaking, (33) may not be the spectral representation of the matrix $\widehat{G}^{(a)}$ because the vectors $\mathbf{v}_{1}^{(a)}(t)$ and $\mathbf{v}_{2}^{(a)}(t)$ are not necessarily orthogonal. Let $\theta(t)=$ $\left|\mathbf{v}_{1}^{(a)}(t) \cdot \mathbf{v}_{2}^{(a)}(t)\right|^{2}$. Then the eigenvalues of $\widehat{G}^{(a)}$ are found to be

$$
\tilde{\lambda}_{1,2}^{(a)}=\frac{1}{2}\left(\lambda_{1}^{(a)}+\lambda_{2}^{(a)}\right) \pm \frac{1}{2} \sqrt{\left(\lambda_{1}^{(a)}-\lambda_{2}^{(a)}\right)^{2}+4 \lambda_{1}^{(a)} \lambda_{2}^{(a)} \theta(t)}
$$

Thus, if at some particular instant one has $\lambda_{1}^{(a)}(t) \approx \lambda_{2}^{(a)}(t)$, then, depending on the details of the dynamics of the eigenvectors, one may have $E_{L}^{(a)} \approx 1$. Strong entanglement is produced when the "weak" component of the OPCM $\sim \lambda_{2}$ is transferred more effectively than the major component $\sim \lambda_{1}$, whose only small part is moved into field $a$ during the evolution. At the same time, as can be seen from (34) after a slight change of notations, such spike of entanglement between particles $a$ is accompanied with disentanglement of particles $b$.

The main condition for this geometric effect is the smallness of the projection of the respective eigenvector of the total OPCM. As a result, the characteristic feature of the OPCM of strongly entangled states in this case is 
$\operatorname{Tr}\left[\widehat{G}^{(a)}\right] \ll \operatorname{Tr}[\widehat{G}]=\operatorname{Tr}\left[\widehat{G}^{(a)}\right]+\operatorname{Tr}\left[\widehat{G}^{(b)}\right]$. That is, the states with $E_{L}^{(a)} \approx 1$ developed from the states with low total entanglement, are characterized by low excitation, while the strongly excited field, say the field $b$ in the considered example, for which one has $\operatorname{Tr}\left[\widehat{G}^{(b)}\right] \approx \operatorname{Tr}[\widehat{G}]$, remains only weakly entangled.

The fact that entanglement of the specific particles is determined by the projections of the total OPCM may lead not only to increased entanglement of the particles but also to disentanglement. Indeed, if for the OPCM given by (31) with $l=1,2$ at some instant the vectors $\mathbf{v}_{1}(t)$ and $\mathbf{v}_{2}(t)$ belong to different subspaces (say, $\widehat{\Pi}_{a} \mathbf{v}_{1}(t)=\mathbf{v}_{1}(t)$ and $\widehat{\Pi}_{b} \mathbf{v}_{2}(t)=\mathbf{v}_{2}(t)$ ) then entanglement of particles of both types, $a$ and $b$, are zero. One can understand this effect introducing the isospin quantum number, so that one value of isospin corresponds to the particle $a$ and another one stands for the particle $b$. Thus, non-zero total entanglement coexisting with zero entanglement of specific particles can be interpreted as stored in isospin.

These effects are of a general nature and do not depend on the number of particles and may play an important role in the dynamics of entanglement in specific systems, for example, the time dependence of entanglement of initially entangled light in a leaky cavity.

\section{Entanglement Produced by Interaction}

As has been demonstrated above, simple linear coupling of quantum fields is not sufficient for the fields to become entangled. We show in this section that the situation is different when there is an interaction in the system. In order to illustrate some important features of the dynamics of entanglement, we consider evolution the of initially disentangled states in systems whose dynamics preserves populations of one-particle states, so that the Hamiltonian can be written as $\mathscr{H}=\sum_{\kappa} \epsilon_{\kappa} \mathcal{N}_{\kappa}+\sum_{\kappa, \lambda} V_{\kappa, \lambda} \mathcal{N}_{\kappa} \mathcal{N}_{\lambda}$, where $\mathcal{N}_{\kappa}=a_{\kappa}^{\dagger} a_{\kappa}$ is the operator of the number of particles in $\kappa$-th one-particle state, $\epsilon_{\kappa}$ are energies of these states, and $V_{\kappa, \lambda}$ are the matrix elements of the interaction potential.

Such systems demonstrate nontrivial entanglement dynamics while admitting an exact solution. First, we consider a simple example of a system with $2 \mathrm{~d}$ one-particle Hilbert space, which we will refer to as an $S U(2)$ model, then we will generalize this consideration to an arbitrary dimension, $S U(n)$ model.

4.1. SU(2)-Model. In the normal form the Hamiltonian preserving population of individual one-particle states has the form

$$
\begin{aligned}
\mathscr{H}= & \sum_{\kappa=+,-} \epsilon_{\kappa} a_{\kappa}^{\dagger} a_{\kappa}+\sum_{\kappa=+,-} U_{\kappa} a_{\kappa}^{\dagger} a_{\kappa}^{\dagger} a_{\kappa} a_{\kappa} \\
& +U_{+-} a_{-}^{\dagger} a_{+}^{\dagger} a_{+} a_{-} .
\end{aligned}
$$

Here the first two terms describe the internal dynamics of the modes and the last term represents the interaction between the modes. Following the discussion in Section 2 it is convenient to rewrite $\mathscr{H}$ in terms of generators of $\mathfrak{g} \mathfrak{t}(2)$ defined in (18). Consider

$$
\mathscr{H}=E_{0}(\mathscr{N})+\epsilon(\mathscr{N}) \mathscr{J}_{z}+\omega \mathscr{F}_{z}^{2},
$$

where $E_{0}(\mathcal{N})=\mathscr{N} \sum_{\kappa}\left(\epsilon_{\kappa}-U_{\kappa}\right) / 2+\mathcal{N}^{2}\left(U_{+}+U_{-}+U_{+-}\right) / 4$ and $\epsilon(\mathscr{N})=\epsilon_{+}-\epsilon_{-}+\left(U_{+}-U_{-}\right)(\mathcal{N}-1)$ depend on the total number of particles $\mathcal{N}=\mathcal{N}_{+}+\mathcal{N}_{-}$, and

$$
\omega=U_{+}+U_{-}-U_{+-} .
$$

The dynamics governed by Hamiltonian (36) was a subject of numerous studies [50-52]. It is constructive, however, to consider its properties from the perspective of the formalism described in Section 2 and establish a connection with entanglement in the particle picture. In particular, we show that the time evolution of entanglement essentially depends on the structure of the initial disentangled state; if initially the system is in spin coherent state it may evolve into a completely entangled state; however, if the system is initially in Glauber's coherent state (i.e., disentangled in both field and particle pictures) it cannot be completely entangled. Moreover, the structure of maximally entangled states is qualitatively different for these initial states.

From $\left[\mathscr{H}, \mathscr{J}_{z}\right]=0$ it follows that the dynamics of entanglement is subject to the general constraint $\left\langle\mathscr{J}_{z}(t)\right\rangle=$ $\left\langle\mathscr{J}_{z}(0)\right\rangle$. Thus, the variation of entanglement is determined by the change with time of the "transversal" component $J_{\perp}^{2}(t)=\left\langle\mathscr{J}_{x}(t)\right\rangle^{2}+\left\langle\mathscr{F}_{y}(t)\right\rangle^{2}=\left\langle\mathscr{F}_{+}(t)\right\rangle\left\langle\mathscr{J}_{-}(t)\right\rangle$, which is unaffected by rotations around the $z$-axis. This imposes an upper limit on the value of entanglement produced by the interaction, $E_{L}(t) \leq E_{L}^{(\max )}=1-\left(\left\langle\mathscr{J}_{z}\right\rangle / j\right)^{2}$. The symmetry with respect to rotations around the $z$-axis implies that dynamics of entanglement is identical for states related through such rotations. An additional simplification comes from the fact that $\mathscr{H}$ preserves the total number of particles. We use this circumstance restricting the main analysis to the case when states are characterized by a definite number of particles, that is, when the initial state is spin coherent states $|\psi(\mathbf{S})\rangle$. Expansion in terms of creation operators yields

$$
\left\langle\mathscr{J}_{z}(t)\right\rangle=\frac{N}{2}\left(\left|S_{1}\right|^{2}-\left|S_{2}\right|^{2}\right)=j \cos (\beta),
$$

where in the last equality we have used spherical coordinates for $\operatorname{spin}$ coherent states $S_{1}=e^{i \phi / 2} \cos (\beta / 2)$ and $S_{2}=$ $e^{-i \phi / 2} \sin (\beta / 2)$.

In order to find the transversal component $\widetilde{J}_{\perp}(t)=$ $J_{\perp}(t) / j$, we first notice that solutions of the operator equations of motion $\dot{\mathscr{J}}_{ \pm}(t)=i\left[\mathscr{H}, \mathscr{J}_{ \pm}(t)\right]$ can be presented as

$$
\mathscr{J}_{ \pm}(t)=\mathscr{J}_{ \pm} \exp \left( \pm i \epsilon t+i \omega t \pm 2 i \omega \mathscr{J}_{z} t\right) \text {. }
$$

The effect of the $\mathscr{J}_{z}$ term on the initial state while calculating $\left\langle\mathscr{F}_{ \pm}(t)\right\rangle \propto\left\langle\psi(\mathbf{S})\left|\mathscr{F}_{ \pm} \exp \left( \pm 2 i \omega \mathscr{F}_{z} t\right)\right| \psi(\mathbf{S})\right\rangle$ reduces to a rotation of the vector $\mathbf{S}$ at the right end. Thus, we obtain

$$
\begin{array}{r}
\left\langle\mathscr{J}_{+}(t)\right\rangle=N S_{1}^{*} S_{2} e^{i \epsilon t}\left(\left|S_{1}\right|^{2} e^{i \omega t}+\left|S_{2}\right|^{2} e^{-i \omega t}\right)^{N-1} \text { and } \\
\widetilde{J}^{2}=\left.4\left|S_{1} S_{2}\right|^{2}|| S_{1}\right|^{2} e^{i \omega t}+\left.\left|S_{2}\right|^{2} e^{-i \omega t}\right|^{2 N-2}
\end{array}
$$


or in the spherical coordinates $\widetilde{J}^{2}=\sin ^{2}(\beta)\left[1-\sin ^{2}(\beta)\right.$ $\left.\sin ^{2}(\omega t)\right]^{N-1}$. It follows from (40) that for $\beta \neq 0$ or $\pi$ the total angular momentum oscillates with the period $T=\pi / \omega$. Instants $t_{k}=T(k+1 / 2)$ with integer $k$ correspond to minimal $\widetilde{J}$ and, hence, maximal entanglement reached from the particular initial state.

In order to better understand the effect of the number of particles on the time evolution of entanglement we consider the case $\beta=\pi / 2$, that is, the case of initially disentangled states with symmetrically populated modes $\left(\left\langle\psi\left|\mathscr{F}_{z}\right| \psi\right\rangle=\right.$ $0)$. Up to rotation around $z$-axis these are the only states (among initially disentangled ones) that yield maximum entanglement $E_{L}=1$ in the course of time evolution. Such states are the object of special interest in our consideration and, therefore, it is worth noting that they constitute a manifold (defined by $\left\langle\psi\left|\mathscr{F}_{z}\right| \psi\right\rangle=0$ ) $\mathscr{M}_{0}$ in the Fock space of all states, including superposition of states with different total number of particles.

From (40) one finds that for these states

$$
\widetilde{J}^{2}=\cos ^{2 N-2}(\omega t) .
$$

The time dependence of entanglement is particularly simple in this case. When the number of particles increases, entanglement considerably changes (practically from 1 to 0 and back) within vicinities of $k T$ with $k=0,1, \ldots$. When the number of particles increases these regions narrow down. In the limit $N \gg 1$ one can approximate

$$
\widetilde{J}^{2}(t) \approx \sum_{n=0}^{\infty} \exp \left[-(N-1)(\omega t-\pi n)^{2}\right] .
$$

Thus, away from the points where $\widetilde{J}(t)=0$, it can be regarded as a train of Gaussian bumps. As a result, when $\sqrt{N} \gg 1$ one can consider the system as spending most of the time in states with high entanglement, $E_{L} \approx 1$.

The Gaussian decay of $\widetilde{J}(t)$ is similar to the Gaussian decay of coherence of central system $[53,54]$ : two spins $1 / 2$ coupled to the bath. In particular, the same dependence of the decay rate on the number of particles $\propto \sqrt{N}$ in the environment [notice $N-1$ in (42)] should be emphasized. There are, however, two important differences between this situation and our case. First, the Gaussian decay for the case of central system appears in the limit of slow dynamics of the environment. In the opposite limit, the decay follows the Lorentzian law and in the intermediate case both types of decays present at different time scales [55]. For the two-mode boson field it is meaningless to separate particular particles and environment due to indistinguishability; however, it is worth noting that $\widetilde{J}(t)$ does not depend on the single-particle energies. The second important difference is that the concurrence of the two-mode boson field exhibits oscillations while the loss of coherence of the central system is irreversible.

Despite the complex structure of the manifold of all completely entangled states, the states reached in the course of evolution of spin coherent states are simple and belong to the class of NOON states. As a result, we can describe these states completely despite the circumstance that the general problem of the state structure refers to the full hierarchy of many-particle density matrices and therefore is not suited for the one-particle approach developed in Section 2 (see, e.g., [56]).

First we notice that due to $\left[\mathscr{H}, \mathscr{F}_{z}\right]=0$ the structure of maximally entangled states is determined solely by a nonlinear term in the Hamiltonian, which we consider in detail and we discuss the effect of the linear terms later. In order to present the state $\left|\widetilde{\Psi}\left(t_{0}\right)\right\rangle=\exp \left\{-i \pi \mathcal{F}_{z}^{2} / 2\right\}|\psi(\mathbf{S})\rangle$ as a NOON state we rewrite

$$
\begin{aligned}
\exp \left\{-i \pi \mathscr{F}_{z}^{2} / 2\right\}= & \exp \left\{-i \frac{\pi}{2}\left(\frac{3}{2} \mathscr{N}\right)^{2}-i \frac{3 \pi}{2} \mathcal{N}_{z}\right\} \\
& \times \exp \left\{-i \frac{\pi}{2} \mathscr{P}^{2}\right\},
\end{aligned}
$$

where $\mathscr{P}=\mathscr{N}_{+}+2 \mathcal{N}_{-}=3 \mathscr{N} / 2-\mathscr{J}_{z}$. A rationale behind such reformulation, which allows one to treat cases of even and odd $N$ on the equal ground $[51,57]$, will be provided in the next subsection.

The operator $\mathscr{P}$ is represented on the basis of population numbers as an integer number $P$. Hence, the action of the last operator on the right in (43) is reduced to a multiplication by a phase factor $e^{-i \pi P^{2} / 2}$, a function of an integer variable with the period $\Delta P=2$. Thus, using the discrete Fourier transform, we obtain

$$
\begin{aligned}
e^{-i \pi \mathscr{P}^{2} / 2}|\psi\rangle & =\sum_{N_{+}, N_{-}} \psi_{N_{+}, N_{-}} \exp \left\{-i \frac{\pi}{2} P^{2}\left(N_{+}, N_{-}\right)\right\}\left|N_{+}, N_{-}\right\rangle \\
& =\frac{1}{\sqrt{n}} \sum_{l=0}^{1} \sum_{N_{+}, N_{-}} \psi_{N_{+}, N_{-}} f_{l} e^{-i(2 \pi / 2) P\left(N_{+}, N_{-}\right) l}\left|N_{+}, N_{-}\right\rangle \\
& =\frac{1}{\sqrt{n}} \sum_{l=0}^{1} f_{l} e^{-i \pi \mathscr{P l}}|\psi\rangle,
\end{aligned}
$$

where $f_{l}=2^{-1 / 2} \sum_{P=0}^{1} \exp \left(i \pi l P-i \pi P^{2} / 2\right)=e^{i \pi l^{2} / 2-i \pi / 4} / \sqrt{2}$.

Expressing $\mathscr{P}$ in terms of $\mathcal{N}$ and $\mathscr{F}_{z}$, one can see that at $t=t_{n}$ the initial spin coherent state turns into a superposition of two spin coherent states related to each other through rotation by $\pi$ around the $z$-axis. For example, if the initial state belongs to $\mathscr{M}_{0}$ then the coordinate system can be chosen in such a way so that the maximally entangled state can be written as $\left(|\mathbf{x}\rangle+e^{i \phi}|-\mathbf{x}\rangle\right) / \sqrt{2}$, or, choosing the $x$-axis as the new quantization axis, as $\left(|N, 0\rangle+e^{i \phi}|0, N\rangle\right) / \sqrt{2}$. The particular orientation of the NOON state and the phase $\phi$ are determined by the terms linear in $\mathscr{F}_{z}$ in (36) and (43) and by the number of particles.

Two important circumstances should be emphasized with this regard. First, while the time dependence of entanglement is a periodic function, however, the time dependence of the state, generally speaking, is not periodic. For example, at $t=$ $2 t_{0}$ entanglement vanishes, and therefore the system returns to a spin coherent state. The form of the state, however, is not necessarily the same as of the initial state but is given by

$$
\left|\Psi\left(2 t_{0}\right)\right\rangle=\exp \left\{-i \mathcal{F}_{z}\left[2 t_{0} \epsilon(\mathcal{N})+3 \pi \mathcal{N}\right]\right\}|\psi\rangle ;
$$


that is, it is rotated around the $z$-axis. Second, the angle of rotation depends on the number of particles, unless $\left(U_{+}-\right.$ $\left.U_{-}\right) / \omega=2 k+1$ with an integer $k$. As a result, states with a different number of particles pass through different sequences of spin coherent states.

This suggests that the time evolution of entanglement of states, which cannot be characterized by a definite number of particles, demonstrates features peculiar from the perspective of the dynamics of the spin coherent states. A general form of a disentangled state is given by (12). Using that $\left[\mathcal{N}, \mathscr{J}_{i}\right]=0$ we find

$$
\left\langle\mathscr{J}_{i}\right\rangle=\sum_{N=0}^{\infty} \frac{\left|\phi_{N}\right|^{2}}{N !}\left\langle\mathscr{J}_{i}(t)\right\rangle_{N},
$$

where $\left\langle\mathscr{J}_{i}(t)\right\rangle_{N}=\left\langle\psi_{N}(\mathbf{S})\left|\mathscr{F}_{i}(t)\right| \psi_{N}(\mathbf{S})\right\rangle$ is the average taken within the $N$-particle sector. Despite an independent contribution of each sector into the average components of the angular momentum, their effect on entanglement is more complex due to $\left|\left\langle\mathscr{F}_{i}(t)\right\rangle\right|^{2}=\sum_{N, N^{\prime}}\left|\phi_{N} \phi_{N^{\prime}}\right|^{2}\left\langle\mathscr{J}_{i}(t)\right\rangle_{N}$ $\left\langle\mathscr{J}_{i}^{\dagger}(t)\right\rangle_{N^{\prime}}$. For concreteness, we limit ourselves to considering a case of special interest when the initial state is disentangled within both field and particle pictures. That is $|\psi\rangle$ is Glauber's coherent state and, hence, $\left|\phi_{N}\right|^{2}=$ $\exp (-\langle N\rangle)\langle N\rangle^{N}$, where $\langle N\rangle$ is the average number of particles. Moreover, we restrict ourselves to the case when $|\psi\rangle \epsilon$ $\mathscr{M}_{0}$ and obtain for the normalized angular momentum $\widetilde{J}=$ $2\left|\left\langle\mathscr{J}_{+}\right\rangle\right| /\langle\mathcal{N}\rangle$

$$
\widetilde{J}(t)=\exp \{-\langle N\rangle[1-\cos (\Delta U t) \cos (\omega t)]\},
$$

where $\Delta U=U_{+}-U_{-}$.

First of all, it should be noted that the maximal value of entanglement is limited from above by $E_{L}^{(\max )}=1-\widetilde{J}_{\min }^{2}$, where $\widetilde{J}_{\min }=\exp (-2\langle N\rangle)$. Thus, Glauber's coherent states do not pass through completely entangled states. However, the "dephasing" of dynamics within sectors corresponding to a different total number of particles brings interesting features to the time dependence of entanglement.

It follows from (47) that, unless $\Delta U / \omega$ is a rational number, entanglement vanishes only at $t=0$. In other words, for a generic interaction potential, the loss of coherence of Glauber's coherent states is irreversible (while, of course, nonmonotonous). The initial loss is described by a Gaussian law $\widetilde{J}^{2}(t) \approx \exp \left(-t^{2} / \tau_{C}^{2}\right)$ with $\tau_{C}^{2}=2 /\langle N\rangle\left(\Delta U^{2}+\omega^{2}\right)$. This expression demonstrates that for the case of superposition of states with different numbers of particles, one has two cooperating channels of entanglement: the internal dynamics within each sector and the dephasing between different sectors.

Another manifestation of existence of an additional channel is the formation of entangled states even if $\omega=0$, when spin coherent states remain disentangled. In this case the variation of entanglement is due to $\Delta U \neq 0$, that is, due to dephasing of states with different number of particles. It follows from (47) that the maximal entanglement in this case is reached at $t=\pi / \Delta U k$ with $k=1,2, \ldots$. It can be seen that this corresponds to the "antiferromagnetic" arrangement of spin coherent states with different total number of particles

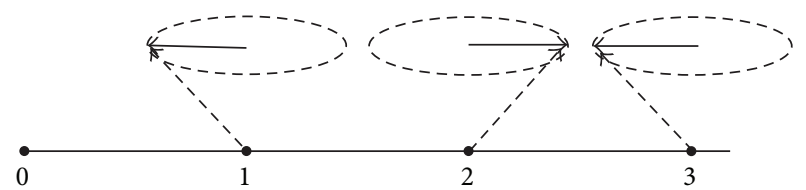

FIGURE 1: Schematic depiction of arrangement of one-particle states within sectors with $N=0,1,2,3$ in maximally entangled state achieved from initial Glauber's coherent state. Dashed arrows show one-particle states represented as unit vectors in $\mathbb{R}^{3}$ and solid arrows show their projections onto $(x, y)$-plane. If the initial state corresponds to $\left\langle\mathscr{F}_{z}\right\rangle=0$, the one-particle states lie in the $(x, y)$ plane.

(see Figure 1). The one-particle states in sectors with the numbers of particles differing by 1 are characterized by opposite transversal components of the average angular momentum.

Clearly, the same result holds when $\omega \neq 0$ but $\Delta U=0$. The instant when NOON states are formed for each component with the definite number of particles (when $\cos (\omega t)=0$ ) does not correspond to maximal entanglement because of the uncompensated angular momentum of the one-particle component. In fact, $E_{L}^{(\max )}$ is reached at $\cos (\omega t)=-1$, when again one has spin coherent states within different sectors but states with odd numbers of particles are oppositely oriented, compared to orientation determined by the linear dynamics.

It follows from this consideration that when both channels contribute, in order to reach $E_{L}^{(\max )}$ it is necessary to have commensurate $\omega$ and $\Delta U$. Thus, for a generic interaction, potential states with maximal entanglement are inaccessible and for $t>0$ the inequality $0<E_{L}(t)<E_{L}^{(\max )}$ holds. The overall dependence of $\widetilde{J}(t)$ on $\Delta U$ and $\omega$ has a simple structure (see Figure 2). In order to describe its main features we introduce $t^{\prime}=\omega t$ and consider the normalized angular momentum as a function of two variables $\widetilde{J}\left(\alpha, t^{\prime}\right)$, where $\alpha=$ $\Delta U / \omega$. The function has a lattice of resonances $\widetilde{J}\left(\alpha=p / k, t^{\prime}=\right.$ $\pi k)=1$, where $p$ and $k$ are integers of the same parity. The resonances are of the Gaussian form with the principal axes along vectors $v_{1}=(1,-x) / \sqrt{1+x^{2}}$ and $v_{2}=(x, 1) / \sqrt{1+x^{2}}$ on the $\left(\alpha, t^{\prime}\right)$-plane and the respective decay parameters $\lambda_{1}=$ $1 / \sqrt{1+x^{2}}$ and $\lambda_{2}=\pi k \sqrt{1+x^{2}}$, where $x=p / \pi k^{2}$ and we have neglected corrections of the order $1 / \pi^{2} k^{2}$.

We would like to emphasize that the reason why Glauber's coherent states cannot be completely entangled is that the dynamics governed by Hamiltonian (36) is unable to cancel the contribution of the one-particle component when the distribution of amplitudes $\phi_{N}$ corresponds to Glauber's coherent state. If one discards the requirement for the initial state to be disentangled in the field picture, it is not difficult to construct such sets of $\phi_{N}$, which produce states evolving through completely entangled states of various structures.

4.2. SU(n)-Model. A general case of $n$-dimensional oneparticle Hilbert space can be treated using the same ideas as those that have been applied in the previous subsection. As has been discussed in Section 2, the most convenient framework for studying entanglement in the particle picture is provided by the language of Lie algebras of Lie 


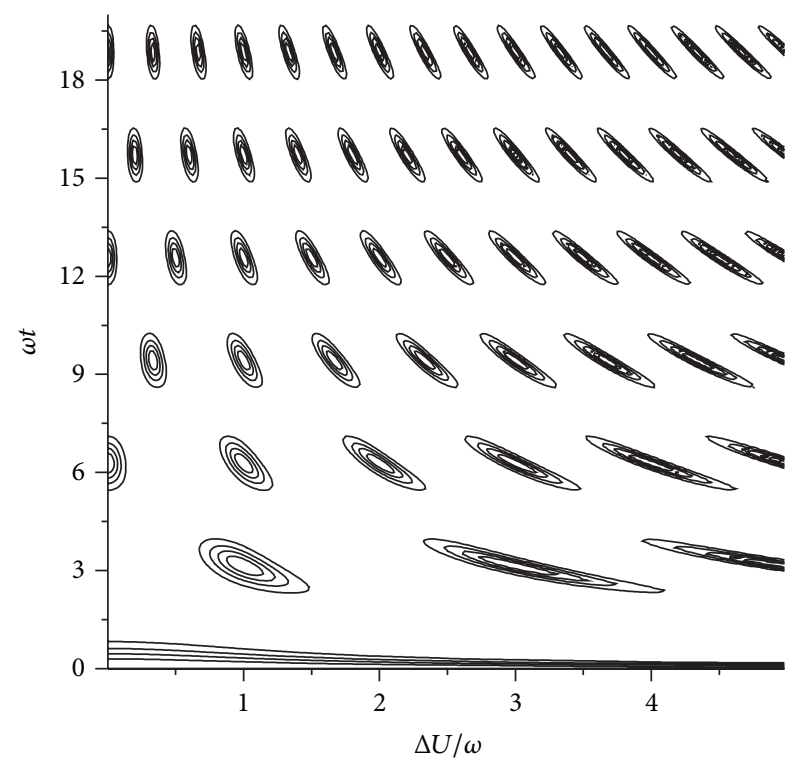

Figure 2: The transversal component of normalized angular momentum $\widetilde{J}=2\left|\left\langle\mathscr{J}_{+}\right\rangle\right| /\langle\mathcal{N}\rangle$ as a function of time and $\Delta U / \omega$ for $\langle\mathcal{N}\rangle=5$. Resonances correspond to points where the initial coherence is restored, $\widetilde{J}=1$.

groups describing transformations of the basis in one-particle Hilbert space. From this perspective it would be, perhaps, more natural to approach the problem in the spirit of studies of dynamical symmetry and to present the Hamiltonian in a form emphasizing the role of $\mathfrak{G} \mathfrak{i t}(n)$ as a spectrum generating algebra [58], for example, in terms of Cartan generators (the elements of the Cartan subalgebra) of $\mathfrak{g} \mathfrak{t}(n)$. We, however, find it more convenient to assume that a basis in the Hilbert space is chosen in such a way that the Hamiltonian can be written solely in terms of the operators of the number of particles in individual modes

$$
\mathscr{H}=\sum_{\kappa} \epsilon_{\kappa} \mathcal{N}_{\kappa}+\sum_{\kappa, \lambda} V_{\kappa, \lambda} \mathcal{N}_{\kappa} \mathcal{N}_{\lambda}
$$

Hamiltonians of this form emerge from a general Hamiltonian with two-particle interaction, when there are $n$ linearly independent one-particle integrals of motion. This corresponds to an off-resonant dynamics of many-level atoms in a cavity [59]. A similar situation may take place in highly symmetrical molecular systems $[60,61]$ and the special interest the $S U(n)$ model presents in the context of the BoseHubbard model [62], where it corresponds to the strong coupling limit and describes fast on-site dynamics.

The structure of Hamiltonian (48) allows one to find the evolution of time with a general ladder operator

$$
\mathscr{J}(\boldsymbol{\mu}, \boldsymbol{\nu})=a_{\mu_{1}}^{\dagger} \cdots a_{\mu_{q}}^{\dagger} a_{\nu_{1}} \cdots a_{\nu_{q}}
$$

where $q$ is the order of the operator. Indeed, noticing that

$$
\left[\mathcal{N}_{\kappa}, \mathscr{J}(\boldsymbol{\mu}, \boldsymbol{\nu})\right]=\mathscr{J}(\boldsymbol{\mu}, \boldsymbol{\nu}) \sum_{j=1}^{q}\left(\delta_{\kappa, \mu_{j}}-\delta_{\kappa, v_{j}}\right)
$$

we find the solution of $\dot{\mathscr{J}}=i[\mathscr{H}, \mathscr{J}]$ in the form

$$
\mathscr{J}(\boldsymbol{\mu}, \boldsymbol{v} ; t)=\mathscr{J}(\boldsymbol{\mu}, \boldsymbol{\nu}) \exp \{i t[\epsilon(\boldsymbol{\mu}, \boldsymbol{\nu})+\mathscr{Q}(\boldsymbol{\mu}, \boldsymbol{\nu})]\},
$$

where $Q(\boldsymbol{\mu}, \boldsymbol{\nu})=2 \sum_{\kappa} U_{\kappa}(\boldsymbol{\mu}, \boldsymbol{\nu}) \mathcal{N}_{\kappa}, \epsilon(\boldsymbol{\mu}, \boldsymbol{\nu})=\sum_{j}\left[\epsilon_{\mu_{j}}-\epsilon_{v_{j}}+\right.$ $\left.U_{\mu_{j}}(\boldsymbol{\mu}, \boldsymbol{\nu})-U_{v_{j}}(\boldsymbol{\mu}, \boldsymbol{\nu})\right]$ and $U_{\kappa}(\boldsymbol{\mu}, \boldsymbol{\nu})=\sum_{j}\left(V_{\kappa, \mu_{j}}-V_{\kappa, v_{j}}\right)$.

For the analysis of one-particle entanglement, we need to consider a particular case $\mathscr{J}(\mu, v)=a_{\mu}^{\dagger} a_{\nu}$. Its time dependence is given by (51) with

$$
\begin{gathered}
Q(\mu, \nu)=2 \sum_{\kappa}\left(V_{\kappa, \mu}-V_{\kappa, \nu}\right) \mathcal{N}_{\kappa}, \\
\epsilon(\mu, \nu)=\epsilon_{\mu}-\epsilon_{\nu}+V_{\mu, \mu}+V_{\nu, \nu}-2 V_{\mu, \nu} .
\end{gathered}
$$

Initially, a disentangled state with a definite number of particles is a spin coherent state $|\psi(\mathbf{S})\rangle$. Finding $\langle\mathscr{J}(\mu, \nu ; t)\rangle=$ $\langle\psi(\mathbf{S})|\mathscr{J}(\mu, v ; t)| \psi(\mathbf{S})\rangle$ follows the same steps as in the $S U(2)$ case. Operator $\exp (i t Q(\mu, \nu))$ acting on $|\psi(\mathbf{S})\rangle$ turns it into another spin coherent state $|\psi(\mathbf{S}(t))\rangle$ with $S_{\kappa}(t)=$ $S_{\kappa} \exp \left(2 i t\left(V_{\kappa, \mu}-V_{\kappa, \nu}\right)\right)$. Thus, we have for the OPCM

$$
\begin{aligned}
G_{\mu, \nu}(t)= & N S_{\mu}^{*} S_{\nu} e^{i t\left(\epsilon_{\mu}-\epsilon_{\nu}\right)+i t\left(V_{\mu, \mu}-V_{\nu, \nu}\right)} \\
& \times\left[\sum_{\kappa}\left|S_{\kappa}\right|^{2} e^{2 i t\left(V_{\kappa, \mu}-V_{\kappa, \nu}\right)}\right]^{N-1} .
\end{aligned}
$$

This yields

$$
E_{L}(t)=E_{L}^{(\max )}(\mathbf{S})-\eta(t),
$$

where

$$
E_{L}^{(\max )}(\mathbf{S})=\frac{n}{n-1}\left(1-\frac{1}{N^{2}} \sum_{\mu}\left|G_{\mu, \mu}\right|^{2}\right)
$$

and $\eta(t)=\left(n / N^{2}(n-1)\right) \sum_{\mu \neq \nu}\left|G_{\mu, \nu}\right|^{2}$. The term $E_{L}^{(\max )}(\mathbf{S})$ is an integral of motion and is determined by the population of one-particle states in the initial state. It can be expressed in terms of the normalized average values of Cartan generators $E_{L}^{(\max )}=1-(n / 2(n-1)) \sum_{j=1}^{n-1} \widetilde{J}_{j}^{2}$, where $\widetilde{J}_{j}=2\left\langle\mathscr{H}_{j}\right\rangle / N$; obviously this expression does not depend on a particular choice of the defining representation of the Cartan generators. Thus, $E_{L}^{(\max )}(\mathbf{S})$ plays a similar role as $1-\left\langle\mathscr{J}_{z}\right\rangle^{2}$ for the $S U(2)$-model and limits the maximal value of entanglement, which can be reached for a particular initial state. The states of special interest are those that admit complete entanglement. It can be seen that these are states with $\left|S_{\kappa}\right|^{2}=1 / n$ for $\kappa=1, \ldots, n$. In more general terms, these are states for which average values of all Cartan generators vanish. This condition defines a manifold $\mathscr{M}_{0}$ in the Fock space. Thus, for a spin coherent state from $\mathscr{M}_{0}$ we have $E_{L}^{(\max )}(\mathbf{S})=1$.

The variation of entanglement with time is related to $\eta(t)$. It can be expressed in terms of the "transversal" component of the Bloch vector $\eta(t)=\widetilde{J}_{\perp}^{2}(t) n / 2(n-1)$ with $\widetilde{J}_{\perp}^{2}=$ $\sum_{j}\left\langle\mathscr{J}_{j}\right\rangle^{2} 4 / N^{2}$, where the summation runs over the ladder operators of $\mathfrak{g} \mathfrak{t}(n)$ in the Hermitian representation. Using 
the expressions found for the off-diagonal elements of OPCM we find

$$
\eta(t)=\left.\left.\frac{n}{n-1} \sum_{\mu \neq \nu}\left|S_{\mu} S_{\nu}\right|^{2}\left|\sum_{\kappa}\right| S_{\kappa}\right|^{2} e^{2 i t\left(V_{\kappa, \mu}-V_{\kappa, \nu}\right)}\right|^{2(N-1)} .
$$

In particular, we find that for $|\psi(\mathbf{S})\rangle \in \mathscr{M}_{0}$ complete entanglement is reached at instants when

$$
F_{\mu, \nu}(t)=\frac{1}{n} \sum_{\kappa} e^{2 i t\left(V_{\kappa, \mu}-V_{\kappa, \nu}\right)}
$$

vanishes for all $\mu \neq \nu$. It is not difficult to see that not all potentials admit the existence of such instants. Moreover, one can show that in striking contrast to the $S U(2)$ model such potentials constitute a special and not very rich class.

In order to see this we first notice that $F_{\mu, \nu}(t)$ can be seen as matrix elements of a matrix $\widehat{F}=\widehat{X} \widehat{X}^{\dagger}$, where $X_{\mu, v}=$ $\exp \left(2 i t V_{\kappa, \mu}\right) / \sqrt{n}$. The condition of complete entanglement in these terms is simply $\widehat{X} \widehat{X}^{\dagger}=\widehat{1}$. Thus, $\widehat{X}$ must be a symmetric (due to $V_{\kappa, \mu}=V_{\mu, \kappa}$ ) unitary matrix. Next, we notice that the condition $\widehat{X} \widehat{X}^{\dagger}=\widehat{1}$ remains invariant under the symmetry transformation $V_{\kappa, \mu} \rightarrow V_{\kappa, \mu}+\widetilde{v}_{\kappa}+\widetilde{v}_{\mu}$ for an arbitrary set of real $\widetilde{v}_{\kappa}$. This transformation can be used to turn all elements of the first row (and the first column) of $V_{\kappa, \mu}$ to zeros. Finally, we notice that up to permutations of coordinates of the orthonormal vectors constituting the matrix or, equivalently, up to permutations of the vectors themselves, symmetric unitary matrix with elements of the first row equal to $1 / \sqrt{n}$ is unique since it is determined by $n(n-1) / 2$ phase factors satisfying $n(n-1) / 2$ inhomogeneous equations. The matrix elements of such matrix are given by

$$
X_{\mu, \nu}=\frac{1}{\sqrt{n}} \exp \left[\frac{2 \pi i}{n}(\mu-1)(\kappa-1)\right] .
$$

Thus, after a proper enumeration of the basis vectors in one-particle Hilbert space, potentials admitting the complete entanglement are of the form

$$
V_{\kappa, \lambda}=\omega \kappa \lambda+v_{\kappa}+v_{\lambda}
$$

with $\omega \neq 0$. It follows from this expression that for the $S U(n)$ model the manifold of such potentials is parametrized by $n+1$ parameters, while the dimension of the manifold of $n \times n$ symmetric matrices is $n(n+1) / 2$. For $S U(2)$ these dimensions coincide and as a result any interaction potential causes complete entanglement (of states with $\langle\mathscr{J}\rangle=0$ ) except for $V=0$, which corresponds to $V_{++}+V_{--}-2 V_{+-}=0$ with the factor 2 stemming from the difference in definitions of $V_{\kappa, \lambda}$ and $U_{ \pm}$and $U_{+-}$in (35). In the $S U(3)$ case, the manifold of completely entangling potentials has codimension two and so on. Thus, by increasing the dimensionality of the one-particle Hilbert space completely, entangling potentials are rather exceptional, at least when dynamics preserves populations of individual modes.

The time dependence $\eta(t)$ when $n$ increases becomes more complex with a characteristic self-similar pattern emerging (see Figure 3(a)). Its overall form can be found

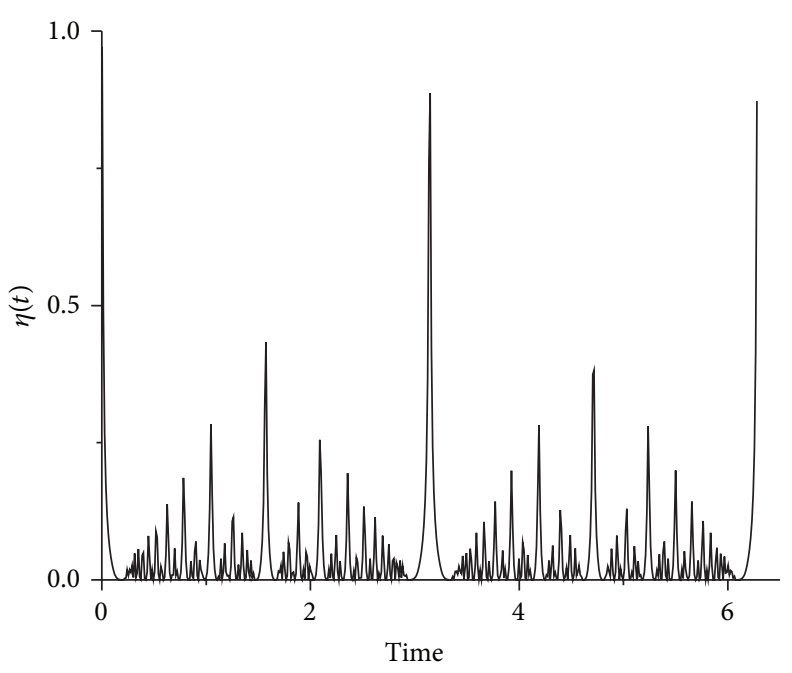

(a)

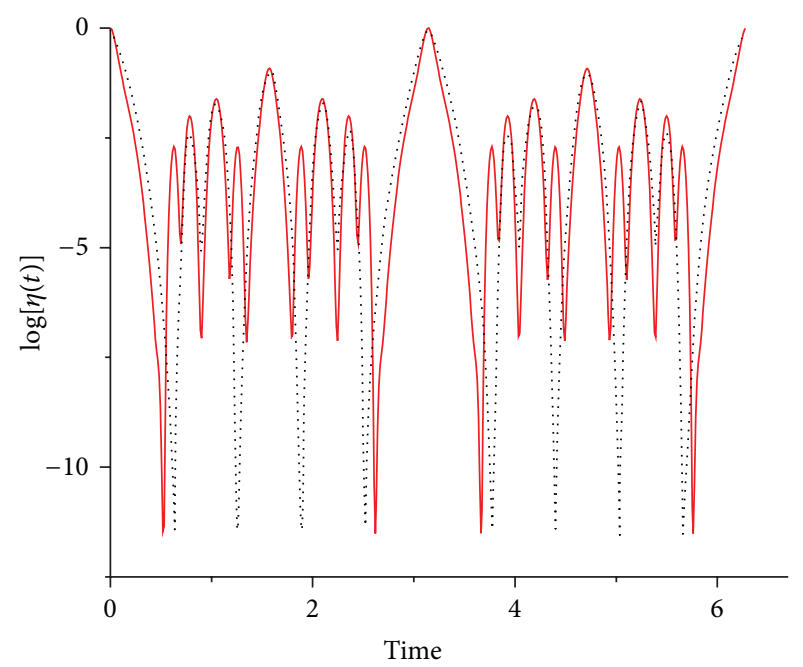

(b)

FIGURE 3: (a) A characteristic pattern of $\eta(t)$ due to partial recoveries of coherence is illustrated for $n=15$ and $N=3$. Time is measured in $V^{-1}$. (b) Dotted and solid lines show $\eta(t)$ for $n=5$ (prime number) and $n=6=3$ !, respectively. This demonstrates that the number of times entangled states are encountered within one period depends on factorization properties of $n$.

using (59) in (56) for $\left|S_{\mathcal{K}}\right|^{2}=1 / n$. In the $S U(2)$, the time dependence of $\widetilde{J}_{\perp}^{2}(t)$ has a typical form of strong (when $N \gg 1$ ) "resonances" at instants $t_{k}=2 \pi k / \omega$, where the one-particle coherence is briefly restored. In the $S U(n)$ case, additional instants of partial recovery of coherence emerge. By performing summation over $\kappa$ in (56) we obtain

$$
\eta(t)=\frac{1}{n(n-1)} \sum_{\mu \neq \nu}\left[\frac{\sin (n t \omega(\mu-\nu))}{n \sin (t \omega(\mu-\nu))}\right]^{2(N-1)} .
$$

When $N \gg 1$ the term under the sum is small unless $\sin (t \omega(\mu-v))=0$. Thus, we have a partial recovery of coherence, when $t=t_{k, p}=\pi k / \omega p$ with coprime $1<$ $p<n$ and $1 \leq k<p$. It follows then that the number of 
such points is equal to the number of irreducible fractions of the form $k / p$ and the degree of recovery (the magnitude $\left.\eta\left(t_{k, p}\right)\right)$ is proportional to the number of pairs $(\mu, \nu)$ satisfying $\sin \left(t_{k, p} \omega(\mu-\nu)\right)=0$.

Of more general interest is the question of initial formation of entanglement. In the limit $N \gg 1$, we obtain for small $t$

$$
\eta(t) \approx \frac{n}{n-1} \sum_{\mu \neq \nu} \exp \left\{-\frac{1}{3} N\left(n^{2}-1\right)(\mu-v)^{2} \omega^{2} t^{2}\right\}
$$

Thus, one has a spectrum of entanglement times (the same holds also for points of partial recovery of coherence). Estimating the entanglement time as the longest one we obtain $\tau_{1}=\sqrt{3 / \omega N\left(n^{2}-1\right)}$.

The distribution of instants when complete entanglement is reached is also more complex compared to the $S U(2)$ case. Within the period $T=\pi / \omega$, one has multiple instants where $\eta(t)=0$. We denote that $t_{1}=\pi / n \omega=T / n$ at the earliest time when the complete entanglement is reached. One can see from (60) that $\eta\left(t_{s}\right)=0$, where $t_{s}=s t_{1}$ with integral $1 \leq s<n$ such that $\operatorname{gcd}(s, n)=1$. From the perspective of determining potentials admitting complete entanglement, this corresponds to permutations of either coordinates of the vectors constituting symmetric unitary matrix $\widehat{X}$ or the vectors themselves.

Thus, the number of points $0<t<T$, when $\eta(t)=0$, depends on prime factorization of $n$. For example, if $n$ is prime, $\eta\left(t_{s}\right)=0$ for all $s=1, \ldots, n-1$. For composite $n$ 's, some of these points are excluded and one has the least density of points of complete entanglement when $n$ is a factorial. It should be noted, however, that $s=1$ and $s=n-1$ always correspond to complete entanglement (see Figure 3(b)).

Finally, we turn to the question of the structure of completely entangled states obtained in the course of evolution of initial spin coherent states. The same approach used for the $S U(2)$ model can be applied here. First, we present the potential as in (59) with $\omega=\pi / n t_{0}$, where $t_{0}$ is the earliest time when a completely entangled state is reached, and study the structure of $\left|\Psi\left(t_{0}\right)\right\rangle=\exp \left(-i \sum_{\kappa} \widetilde{\epsilon}_{\kappa} \mathcal{N}_{\kappa}-i \pi \mathscr{P}^{2} / n\right)|\psi(\mathbf{S})\rangle$, where $\widetilde{\epsilon}_{\kappa}=\epsilon_{\kappa}+\mathcal{N} v_{\kappa}$ and $\mathscr{P}=\sum_{\kappa} \kappa \mathcal{N}_{\kappa}$. Similar to what we had in the previous subsection, the structure of the entangled state is determined by the term $\propto \mathscr{P}^{2}$, while the term $\propto \widetilde{\epsilon}_{\kappa}(\mathscr{N})$ affects only the orientation of the state. Therefore, in order to analyze the structure of the state it suffices to consider $\left|\widetilde{\Psi}\left(t_{0}\right)\right\rangle=e^{-i \pi \mathscr{P}^{2} / n}|\psi(\mathbf{S})\rangle$. The idea is the same as for the $S U(2)$-model. We expand $|\psi(\mathbf{S})\rangle$ over basis states on the basis of population numbers and use the fact that action of $\mathscr{P}$ on these states is equivalent to multiplication by an integer number $P$. Thus, $e^{-i \pi \mathscr{P}^{2} / n}$ can be regarded as a function of an integer variable and with the help of the discrete Fourier transform can be expanded in terms of linear operators in $\mathscr{P}$.

The implementation of this Hubbard-Stratonovich type of transformation is slightly different for even and odd $n$, and therefore we consider these cases separately.
For even $n$ the period of $e^{-i \pi P^{2} / n}$ is $\Delta P=n$ and we obtain

$$
\left|\widetilde{\Psi}\left(t_{0}\right)\right\rangle=\frac{1}{\sqrt{n}} \sum_{l=0}^{n-1} f_{l} e^{-i 2 \pi l \mathscr{P} / n}|\psi(\mathbf{S})\rangle
$$

where

$$
f_{l}=\frac{1}{\sqrt{n}} \sum_{P=0}^{n-1} e^{i 2 \pi l P / n-i \pi P^{2} / n}=e^{i \pi l^{2} / n-i \pi / 4}
$$

Here we have used

$$
\sum_{P=0}^{n-1} e^{-i \pi\left(P^{2}+b P\right) / n}=\sqrt{n} e^{-i\left(n-b^{2}\right) / 4 n}
$$

which follows from Siegel's reciprocity relation for generalized quadratic Gauss sums $[63,64]$ and is valid when $n+b$ is even.

When $n$ is odd we need to consider $e^{-i \pi \mathscr{P} / n} e^{-i \pi \mathscr{P}^{2} / n+i \pi \mathscr{P} / n}$ instead. Here the second exponential is a periodic function with the period $\Delta P=n$. Thus, using (64), we find

$$
\left|\widetilde{\Psi}\left(t_{0}\right)\right\rangle=\frac{1}{\sqrt{n}} e^{-i \pi \mathscr{P} / n} \sum_{l=0}^{n-1} f_{l} e^{-i 2 \pi \mathscr{P} l / n}|\psi(\mathbf{S})\rangle,
$$

where $f_{l}=e^{i \pi l(l-1) / n-i \pi(n-1) / 4 n}$

Applying (62) and (65) to the case when $|\psi(\mathbf{S})\rangle \in$ $\mathscr{M}_{0}$ we can see that the structure of completely entangled states is essentially the same as in the $S U(2)$ case: they are superpositions of $n$ spin coherent states characterized by state vectors $\mathbf{S}_{l}=e^{-2 \pi i \widehat{P} l / n} \mathbf{S}$, where $\widehat{P}=\operatorname{diag}(1,2, \ldots, n)$ and $l=0, \ldots n-1$. It can be easily seen that $\mathbf{S}_{l}^{*} \cdot \mathbf{S}_{l^{\prime}}=\delta_{l, l^{\prime}}$. In other words, a completely entangled state is built of spin coherent states corresponding to an orthogonal set of oneparticle states. Thus, completely entangled states belong to the class of NOON states.

The structure of completely entangled states reached at $t=t_{s}$ with $s>1$ differs only by precise relations between phases of superimposed spin coherent states. In order to see this we consider the more transparent case of even $n$. The expansion of $e^{-i \pi s \mathscr{P}^{2} / n}|\psi(\mathbf{S})\rangle$ is given by (62) with amplitudes

$$
f_{l}^{(s)}=e^{i \pi s k_{l}^{2} / n} \frac{1}{\sqrt{n}} \sum_{P=0}^{n-1} e^{-i \pi s P^{2} / n}
$$

where $k_{l}$ is defined by $l \equiv s k_{l}(\bmod n)$ or $k_{l} \equiv s^{n-1} l(\bmod n)$. In (66) only the phase factor in front of the sum is relevant and it describes the difference between completely entangled states reached at different $t_{s}$.

The obtained results can be generalized to the case of superpositions of states with different total numbers of particles following the same line as for the $S U(2)$ model. For an initially disentangled state, presented as in (12), we have

$$
\langle\mathscr{J}(\mu, v ; t)\rangle=\sum_{N=0}^{\infty} \frac{\left|\phi_{N}\right|^{2}}{N}\langle\mathscr{J}(\mu, v ; t)\rangle_{N}
$$


Thus, one can expect a qualitative similarity between $S U(2)$ and $S U(n)$ models. Let the initial state be Glauber's coherent state characterized by the one-particle state $\mathbf{S}$ and the average total number of particles $\langle N\rangle$. Then, the time dependence of entanglement is given by (54) with

$$
\begin{aligned}
\eta(t)= & \frac{n}{n-1} e^{-2\langle N\rangle} \\
& \times \sum_{\mu \neq \nu}\left|S_{\mu} S_{\nu}\right|^{2} \exp \left\{\sum_{\kappa}\left|S_{\kappa}\right|^{2} \cos \left(2 t\left(V_{\kappa, \mu}-V_{\kappa, \nu}\right)\right)\right\} .
\end{aligned}
$$

First of all it should be noted that $\eta(t)>0$; thus complete entanglement cannot be achieved for any interaction potential. Instead one has to consider the problem of the minimal value that $\eta(t)$ can reach for given potential and initial state. This complex problem is significantly simplified for the case when the interaction potential and the initial state admit complete entanglement within sectors with a definite number of particles, that is, when potential can be presented as in (59) and $\left|S_{\kappa}\right|^{2}=1 / n$. In this case we have

$$
\begin{aligned}
\eta(t)=\frac{1}{n(n-1)} \sum_{\mu \neq \nu} \exp \{-2\langle N\rangle[ & -\cos \left(\Delta U_{\mu, \nu} t\right) \\
& \left.\left.\times \frac{\sin [n t \omega(\mu-\nu)]}{n \sin [t \omega(\mu-\nu)]}\right]\right\},
\end{aligned}
$$

where $\Delta U_{\mu, \nu}=2\left(v_{\mu}-v_{\nu}\right)+\omega(\mu-v)(n+1)$. This equation has the same structure as (47) and posseses the same basic features related to the existence of different channels of entanglement in the case of superposition of states with different number of particles. One channel is through the formation of NOON states as has been discussed above and another channel is related to dephasing of states belonging to sectors with a different total number of particles. As well as in the $S U(2)$ case, initially, at $t \approx 0$, the effects of these two mechanisms combine leading to faster entanglement compared to the case of spin coherent state.

When $\Delta U_{\mu, \nu} \equiv 0$ there is an interesting dependence of the general profile of $\eta(t)$ on the parity of $n$. In order to see it, we consider the case $\langle N\rangle \gg 1$. The magnitude of $\eta(t)$ in this case is determined by the largest term in the sum over $\mu$ and $\nu$. Therefore, a special attention should be paid to vicinities of $t_{p, k}$, instants of partial recovery of coherence. The respective contributions to $\eta(t)$ have the form $\exp \left\{-2\langle N\rangle\left[1-(-1)^{(n+1) k}\right]\right\}$ with integral $k$. Thus, when $n$ is odd all $t_{p, k}$ correspond to partial recovery of coherence. While for even $n$, some of these points in fact correspond to increased entanglement, an effect similar to what we have seen in the $S U(2)$ case (see Figure 4).

When both entangling channels contribute (i.e., we have $\omega \neq 0$ and $\left.\Delta U_{\mu, \nu} \neq 0\right)$, the initial time dependence of entanglement is relatively simple: the contribution of terms with $\Delta U_{\mu, v} \neq 0$ vanishes at time scales $\sim 1 / \Delta U_{\mu, v}$, leading to a reduction of entanglement oscillations. The long time behavior, however, is quite complex and strongly depends on the commensurability of $\Delta U_{\mu, \nu}$ and $\omega$.

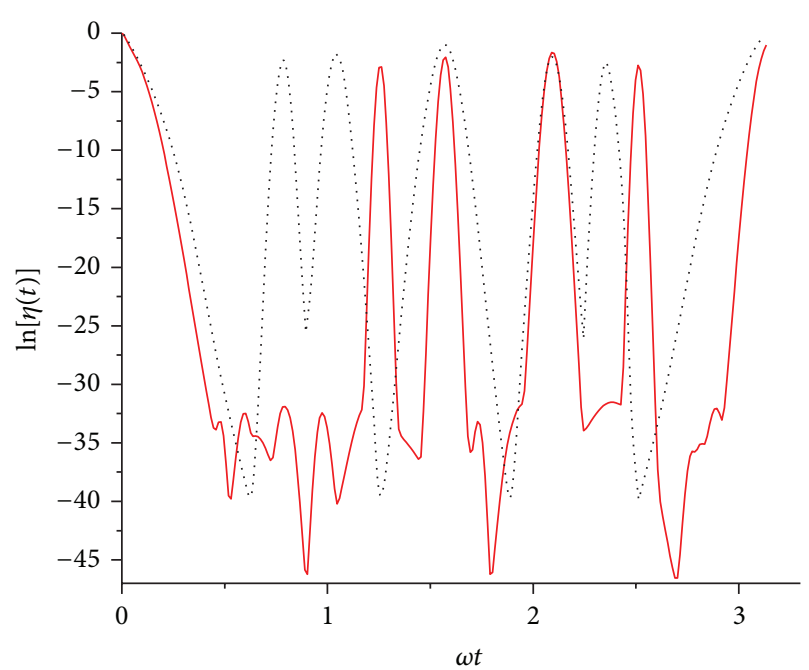

FIgURE 4: Variation of $\eta(t)$ for initially Glauber's state with the parity of $n$ : solid and dotted lines correspond to $n=6$ and $n=$ 5 , respectively, for $N=20$. When only one entangling channel contributes (i.e., $\Delta U_{\mu, v} \equiv 0$ in (69)), stronger entanglement can be reached in $S U(n)$ systems with even $n$.

\section{Entanglement Dynamics in Two-Mode Jaynes-Cummings Model}

In the previous sections we have considered only interaction between boson fields. Here, we extend the analysis for the case when the boson field interacts with the system, whose dynamics is subject to the Pauli exclusion principle. More specifically, we consider the evolution of entanglement of photons in a cavity with $M$ quantum dots (QD) modelled by two-level systems admitting transitions characterized by definite helicities.

The excitations of QDs are described by $c_{s}^{\dagger}(\mathbf{r})$, operators creating electron with spin $s$ in the QD at point $\mathbf{r}$, and $v_{s}^{\dagger}(\mathbf{r})$, operators creating holes. A dot in the ground state is excited by creating an electron-hole pair. This is described by acting on the QD's ground state by $v_{s}^{\dagger}(\mathbf{r}) c_{s^{\prime}}^{\dagger}(\mathbf{r})$. The helicity of the respective transition is given by $s-s^{\prime}$. The operators dealing with transitions of fixed helicity constitute $\mathfrak{g} \mathfrak{t}(2)$ algebras and, therefore, it is more convenient to introduce the respective generators: $\mathcal{S}_{ \pm}^{+}(\mathbf{r})=v_{\mp 3 / 2}^{\dagger}(\mathbf{r}) c_{\mp 1 / 2}^{\dagger}(\mathbf{r}), \mathcal{S}_{ \pm}^{-}(\mathbf{r})=$ $c_{\mp 1 / 2}(\mathbf{r}) v_{\mp 3 / 2}(\mathbf{r})$, and so on, where we have assumed that the states in the valence bands are heavy-holes.

Using these notations, we present the Hamiltonian governing the dynamics of the system as a Hamiltonian of twomode Jaynes-Cummings model $[65,66]$. Consider

$$
\mathscr{H}=\sum_{\kappa} \epsilon_{\kappa} a_{\kappa}^{\dagger} a_{\kappa}+\sum_{\kappa, i} v_{\kappa}\left(\mathbf{r}_{i}\right) \mathcal{S}_{\kappa}^{z}\left(\mathbf{r}_{i}\right)+\mathscr{H}_{\text {int }}
$$

where $i$ runs over QDs and $\nu_{\kappa}(\mathbf{r})$ are the excitation energies of QDs and $\mathcal{S}_{\kappa}^{z}(\mathbf{r})=\left[\mathcal{S}_{\kappa}^{+}(\mathbf{r}), \mathcal{S}_{\kappa}^{-}(\mathbf{r})\right] / 2$. In terms of the electron and hole operators, $\mathcal{S}_{\kappa}^{z}(\mathbf{r})$ are written 
as $\mathcal{S}_{ \pm}^{z}(\mathbf{r})=\left[c_{\mp 1 / 2}^{\dagger} c_{\mp 1 / 2}+v_{\mp 3 / 2}^{\dagger} v_{\mp 3 / 2}-1\right] / 2$. The interaction between photons and QDs is given by

$$
\mathscr{H}_{\mathrm{int}}=\sum_{\kappa, i}\left[\omega_{\kappa}\left(\mathbf{r}_{i}\right) \mathcal{S}_{\kappa}^{+}\left(\mathbf{r}_{i}\right) a_{\kappa}+\omega_{\kappa}^{*}\left(\mathbf{r}_{i}\right) \mathcal{S}_{\kappa}^{-}\left(\mathbf{r}_{i}\right) a_{\kappa}^{\dagger}\right]
$$

where $\omega_{\kappa}(\mathbf{r})$ are the respective Rabi frequencies. Generally speaking, they are complex numbers depending on the phase of the photon mode. Their argument, however, is of minor importance and, therefore, in what follows we will assume that $\omega_{\kappa}(\mathbf{r})=\omega_{\kappa}^{*}(\mathbf{r}) \geq 0$.

We do not require the parameters of the QDs to be the same. This accounts for the variation of QD's excitation energies due to the size fluctuations and the variation of the Rabi frequencies due to position of the QDs in the cavity. The dynamics of entanglement in this system is a result of interference of harmonics with incommensurate frequencies [67] and in the long time limit the time dependence of entanglement is very complex. However, when the number of photons is large, then for relatively short time scales the evolution of entanglement demonstrates universal features, which we will analyse in detail. More specifically, we consider the evolution of entanglement for the case when initially we have a disentangled photon state in the cavity and all QDs are in the ground state. This closely corresponds to the situation when, for example, the cavity in the ground state is pumped by an external source.

It is convenient to take into account that the total number of excitations remains constant: $\left[\mathscr{H}, \mathcal{N}_{\kappa}\right]=0$, where $\mathcal{N}_{\kappa}=a_{\kappa}^{\dagger} a_{\kappa}+\sum_{i}\left(\mathcal{S}_{\kappa}^{z}\left(\mathbf{r}_{i}\right)+1 / 2\right)$. The effect of the operator $\exp \left(-i t \sum_{\kappa} \epsilon_{\kappa} \mathcal{N}_{\kappa}\right)$ on the dynamics is simple and reduces to rotation of the initial photon state around the $z$-axis. It results in acquiring phase factors by $\left\langle\mathscr{F}_{ \pm}\right\rangle$and, therefore, does not affect the entanglement. This circumstance allows us to exclude the single-photon contribution to the energy and to introduce $\widetilde{\mathscr{H}}=\mathscr{H}-\sum_{\kappa} \epsilon_{\kappa} \mathcal{N}_{\kappa}=\sum_{i, \kappa} \Delta_{\kappa}\left(\mathbf{r}_{i}\right) \mathcal{S}_{\kappa}^{z}\left(\mathbf{r}_{i}\right)+\mathscr{H}_{\text {int }}$, where $\Delta_{\kappa}(\mathbf{r})=\nu_{\kappa}(\mathbf{r})-\epsilon_{\kappa}$ is the detuning of the $\kappa$-transition in the QD at $\mathbf{r}$ from the resonance with the cavity mode.

The nontrivial part of the dynamics is due to the excitation of a QD by absorbing a photon. Let the initial state of the system be characterized by definite numbers of photons with each polarization, $|\Psi(t=0)\rangle=\left|N_{+}, N_{-}\right\rangle|0\rangle_{D}$, where $|0\rangle_{D}$ corresponds to all QDs being their ground states. Then at any instant we can present

$$
|\Psi(t)\rangle=\sum_{\widehat{k}_{+}, \widehat{k}_{-}} \psi_{\widehat{k}_{+}, \widehat{k}_{-}}(t)\left|\widehat{k}_{+}, \widehat{k}_{-}\right\rangle,
$$

where $\left|\widehat{k}_{+}, \widehat{k}_{-}\right\rangle=\left|N_{+}-k_{+}, N_{-}-k_{-}\right\rangle\left|\widehat{k}_{+}, \widehat{k}_{-}\right\rangle_{D}$ and $\left|\widehat{k}_{+}, \widehat{k}_{-}\right\rangle_{D}$ are the QDs state with $k_{ \pm}$QDs in excited \pm -states and $\widehat{k}_{ \pm}$describe the distribution of the excited states over the QDs. The matrix element of the Hamiltonian between the respective states is proportional, for example, to $\left\langle N_{\kappa}-k_{\kappa}^{\prime}\left|a_{\kappa}\right| N_{\kappa}-\right.$ $\left.k_{\kappa}\right\rangle=\delta_{k_{\kappa}^{\prime}, k_{\kappa}} \sqrt{N_{\kappa}-k_{\kappa}}$, which varies in the range from $\sqrt{N_{\kappa}}$ to $\sqrt{N_{\kappa}-M}$. In the limit $N_{\kappa} \gg M$, we can neglect this variation, which is sufficient for the consideration of relatively short time scales, as will be elaborated below. Moreover, if one is interested, for example, only in photon observables (say, the photon OPDM), one can extend this approximation further and neglect the variation of photon states in expansion (72) and set $\left|\widehat{k}_{+}, \widehat{k}_{-}\right\rangle \approx\left|N_{+}, N_{-}\right\rangle\left|\widehat{k}_{+}, \widehat{k}_{-}\right\rangle_{D}$ up to terms $\propto 1 / \sqrt{N_{ \pm}}$. Thus, treating consistently the limit $N_{\kappa} \gg M$ one can approximate $\widetilde{\mathscr{H}}$ by replacing photon creation and annihilation operators by $\sqrt{a_{\kappa}^{\dagger} a_{\kappa}}$, similar to the Bogoliubov approximation in the theory of excitations of the Bose-Einstein condensates. The validity of this approximation for initially disentangled states, however, is not self-evident. Indeed, except the cases when $N_{+}=0$ or $N_{-}=0$, disentangled states are not characterized by a definite numbers of photons. Therefore, generally speaking, the condition $N_{\kappa} \gg M$ is not fulfilled for disentangled states. On the other hand, states with a significantly mismatched number of photons with different polarizations are typical for states with $\left\langle\mathscr{J}_{z}\right\rangle / N \sim 1$, and the time evolution of entanglement of such states is rather simple due to the limitation on the maximum value of entanglement. Similar to previous sections, states of our main interest are those which pass through completely entangled states. These are states with $\left|\left\langle\mathscr{J}_{z}\right\rangle\right| \leq M \ll N$ and for simplicity we will consider states with $\left\langle\mathscr{J}_{z}\right\rangle=0$. The contribution of states with mismatched $N_{+}$and $N_{-}$decreases with $m=\left(N_{+}-N_{-}\right) / 2$ fast $\propto \exp \left[-\left(m / m_{0}\right)^{2}\right]$, where $m_{0} \sim 2\left(\pi j^{5}\right)^{1 / 8}$. Thus, if $j-m_{0} \gg$ $M$, the contribution of the states, for which the condition $N_{\kappa} \gg M$ does not hold, is negligible.

Adopting these approximations we can write the Hamiltonian in a simple form

$$
\widetilde{\mathscr{H}}=\sum_{\kappa, \mathbf{r}, i} \mathscr{B}_{\kappa}\left(\mathbf{r}_{i}\right) \cdot \mathcal{S}_{\kappa}\left(\mathbf{r}_{i}\right)
$$

where $\mathscr{B}_{\mathcal{K}}(\mathbf{r})=\left(\mathcal{S}_{\kappa}^{x}(\mathbf{r}), \mathcal{S}_{\mathcal{K}}^{y}(\mathbf{r}), \mathcal{S}_{\mathcal{K}}^{z}(\mathbf{r})\right)$ with $\mathcal{S}_{\kappa}^{x}(\mathbf{r})=\left[\mathcal{S}_{\mathcal{K}}^{+}(\mathbf{r})+\right.$ $\left.\mathcal{S}_{\mathcal{\kappa}}^{-}(\mathbf{r})\right] / 2$, and so on, and $\mathscr{B}_{\kappa}(\mathbf{r})=\left(2 \omega_{\mathcal{\kappa}}(\mathbf{r}) \sqrt{a_{\mathcal{K}}^{\dagger} a_{\kappa}}, 0, \Delta_{\kappa}(\mathbf{r})\right)$. Hamiltonian (73) has the form of a Hamiltonian of a lattice of spins in a magnetic field depending on the number of photons. It should be noted that "spins" are formed by the generators of the $\mathfrak{g} \mathfrak{u}(2)$ algebras corresponding to each helicity independently.

Different terms in (73) commute and, therefore, the operator of evolution is represented as a product of operators acting on individual QDs. Thus, by expanding the photon state over the basis of population numbers one can evaluate the action of the operator of evolution on the initial state and find the resulting photon OPDM. The final expression turns out to be very cumbersome due to noncommutativity of operators $\mathscr{B}_{\kappa}(\mathbf{r}) \cdot \mathcal{S}_{\kappa}(\mathbf{r})$ taken for photon states with different $N_{\kappa}$. It can be seen, however, that the variation of the directions of $\mathscr{B}_{\kappa}(\mathbf{r})$ leads to corrections to the state $\propto$ $1 / \sqrt{N}$, which were neglected while deriving (73). Thus, we can approximate $\mathscr{B}_{\kappa}(\mathbf{r}) \approx \widehat{\mathbf{e}}_{\kappa}(\mathbf{r}) \sqrt{4 \omega_{\kappa}^{2}(\mathbf{r}) a_{\kappa}^{\dagger} a_{\kappa}+\Delta_{\kappa}^{2}(\mathbf{r})}$, where $\widehat{\mathbf{e}}_{\kappa}(\mathbf{r})=\widetilde{\mathscr{B}}_{\kappa}(\mathbf{r}) / \widetilde{\mathscr{B}}_{\kappa}(\mathbf{r})$ and $\widetilde{\mathscr{B}}_{\kappa}(\mathbf{r})=\left(2 \omega_{\kappa}(\mathbf{r}) \sqrt{\mathscr{J}_{0}}, 0, \Delta_{\kappa}(\mathbf{r})\right)$ with $\mathscr{J}_{0}=\left(a_{+}^{\dagger} a_{+}+a_{-}^{\dagger} a_{-}\right) / 2$. Treating $\mathscr{J}_{z}$ as a small correction to $\mathscr{J}_{0}$ and keeping only the linear term we finally obtain

$$
\widetilde{\mathscr{H}}=\sum_{\kappa, i} \widetilde{\mathscr{B}}_{\kappa}\left(\mathbf{r}_{i}\right) \cdot \mathcal{S}_{\kappa}\left(\mathbf{r}_{i}\right)+\mathscr{J}_{z} \sum_{\kappa, i} \boldsymbol{\Omega}_{\kappa}\left(\mathbf{r}_{i}\right) \cdot \mathcal{S}_{\kappa}\left(\mathbf{r}_{i}\right),
$$


where $\boldsymbol{\Omega}_{\kappa}(\mathbf{r})=2 \widehat{\mathbf{e}}_{\kappa}(\mathbf{r}) h_{\kappa} \omega_{\kappa}^{2}(\mathbf{r}) / \widetilde{\mathscr{B}}_{\kappa}(\mathbf{r})$ with $h_{\kappa}= \pm$ for $\kappa= \pm$. It should be emphasized that all terms entering (74) commute with each other. This yields a compact expression for the photon OPDM. We provide it for the case when the initial photon state has the definite number of particles, so that we can replace $\mathscr{J}_{0} \rightarrow j$ in expressions for $\widetilde{\mathscr{B}}_{\kappa}(\mathbf{r})$ and $\boldsymbol{\Omega}_{\kappa}(\mathbf{r})$. Using the Heisenberg representation we find

$$
\begin{aligned}
\left\langle\mathscr{F}_{+}(t)\right\rangle=\left\langle\mathcal{F}_{+}(0)\right\rangle\left\langle\left. 0\right|_{D} \exp \left[i t \sum_{\kappa, i} \boldsymbol{\Omega}_{\kappa}\left(\mathbf{r}_{i}\right) \cdot \mathcal{S}_{\kappa}\left(\mathbf{r}_{i}\right)\right] \mid 0\right\rangle_{D} \\
=\left\langle\mathscr{F}_{+}(0)\right\rangle \prod_{\kappa, i}\left[\cos \left(\frac{\Omega_{\kappa}\left(\mathbf{r}_{i}\right) t}{2}\right)\right. \\
\left.+i h_{\kappa} \frac{\Delta_{\kappa}\left(\mathbf{r}_{i}\right)}{\widetilde{B}_{\kappa}\left(\mathbf{r}_{i}\right)} \sin \left(\frac{\Omega_{\kappa}\left(\mathbf{r}_{i}\right) t}{2}\right)\right] .
\end{aligned}
$$

First we discuss the case when all QDs are in resonance with the cavity, $\Delta_{\kappa}(\mathbf{r}) \equiv 0$. In this case, $\left\langle\mathscr{F}_{+}(t)\right\rangle=$ $\left\langle\mathscr{F}_{+}(0)\right\rangle \prod_{\kappa, i} \cos \left[\omega_{\kappa}\left(\mathbf{r}_{i}\right) t / 2 \sqrt{j}\right]$. The complete entanglement is reached at instants $T_{\kappa}^{(k)}\left(\mathbf{r}_{i}\right)=\sqrt{j} \pi(1+2 k) / \omega_{\kappa}\left(\mathbf{r}_{i}\right)$ with integer $k$. Thus, we have a spectrum of instants corresponding to complete entanglement. With this regard it should be noted that accounting for terms omitted while deriving (74) leads to significant variations at time scales $\sim j T_{\kappa}^{(1)}(\mathbf{r}) \gg T_{\kappa}^{(1)}(\mathbf{r})$. Thus, the dynamics described by approximate Hamiltonian (74) is sufficient for covering multiple recurrence of completely entangled states.

In order to discuss the structure of completely entangled states we first notice that both $\widetilde{\mathbf{B}}_{\kappa}(\mathbf{r})$ and $\boldsymbol{\Omega}_{\kappa}(\mathbf{r})$ have only $x$-component. Choosing $x$-axis as the new quantization axis we can rewrite (74) as $\widetilde{\mathscr{H}}=\sum_{\kappa, i} \widetilde{B}_{\kappa}\left(\mathbf{r}_{i}\right) \widetilde{\mathcal{S}}_{\kappa}^{z}\left(\mathbf{r}_{i}\right)+$ $\sum_{\kappa, i} h_{\kappa} \Omega_{\kappa}\left(\mathbf{r}_{i}\right) \mathcal{F}_{z} \widetilde{\mathcal{S}}_{\kappa}^{z}\left(\mathbf{r}_{i}\right)$ with the initial state of the QDs being the product of $\left(\left|\mathbf{x}_{\kappa}\left(\mathbf{r}_{i}\right)\right\rangle+\left|-\mathbf{x}_{\kappa}\left(\mathbf{r}_{i}\right)\right\rangle\right) / \sqrt{2}$, where $\delta_{\kappa}^{x}(\mathbf{r}) \mid \pm$ $\left.\mathbf{x}_{\kappa}(\mathbf{r})\right\rangle= \pm(1 / 2)\left| \pm \mathbf{x}_{\kappa}(\mathbf{r})\right\rangle$. The resultant Hamiltonian has the form of a spin lattice Hamiltonian with the Ising-type interaction with photons playing the role of a many-body state at a special, "defect", site of the lattice. A full discussion of completely entangled states in this case requires expanding the scope of the present paper to the lattice case. For our purposes, however, it is sufficient to note that the completely entangled states are a many-body generalization of graph states $[68,69]$ and can be described following the standard procedure. The states are described by a simple graph: the central vertex represents photons and other vertices correspond to different QDs and helicities of the transition. The vertex representing $\mathcal{\kappa}$-transition in the $i$ th $\mathrm{QD}$ gets connected to the photon vertex at instant $T_{\kappa}^{k}\left(\mathbf{r}_{i}\right)$, as is illustrated in Figure 5.

We consider explicitly the simplest case, when the graph has at most two vertices; that is, at instant $t_{0}$ there is at most one set of $\left(\mathbf{r}_{0}, \kappa_{0}, k_{0}\right)$ such that $T_{\kappa_{0}}^{\left(k_{0}\right)}\left(\mathbf{r}_{0}\right)=t_{0}$. We present $\widetilde{\mathscr{H}}=$ $\widetilde{\mathscr{H}}_{0}+\widetilde{\mathscr{H}}^{\prime}$, where $\widetilde{\mathscr{H}}_{0}$ contains only the term with $\kappa=\kappa_{0}$ and $\mathbf{r}=\mathbf{r}_{0}$ from (74) and $\widetilde{\mathscr{H}}^{\prime}$ has the rest of the terms. Next, let

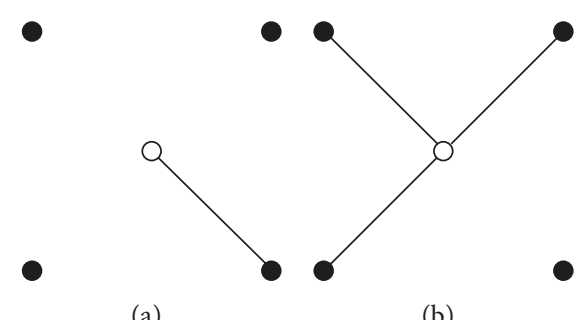

(a)

(b)

FIGURE 5: Schematic depiction of completely entangled state at instant $t$ for the system of four QDs (filled vertices) and photons (opened central vertex): (a) the condition of complete entanglement, $t=T_{\kappa}^{(k)}\left(\mathbf{r}_{i}\right)$ is fulfilled only for one QD; (b) three QDs satisfy the condition of the complete entanglement and; that is, there are three sets of $(i, \kappa, k)$ such that $T_{\kappa}^{(k)}\left(\mathbf{r}_{i}\right)=t$.

the initial photon state be the coherent spin state $|\psi(\mathbf{S})\rangle$, then the completely entangled state can be presented as

$$
\begin{aligned}
\left|\Psi_{0}\right\rangle=e^{-i \widetilde{\mathscr{H}}^{\prime} t_{0}} \frac{1}{\sqrt{2}}\left(|\psi(\widetilde{\mathbf{S}})\rangle\left|\widetilde{\mathbf{x}}_{\kappa_{0}}\left(\mathbf{r}_{0}\right)\right\rangle_{D}\right. \\
\left.+|\psi(-\widetilde{\mathbf{S}})\rangle\left|-\widetilde{\mathbf{x}}_{\kappa_{0}}\left(\mathbf{r}_{0}\right)\right\rangle_{D}\right)\left|0^{\prime}\right\rangle_{D},
\end{aligned}
$$

where $\left|\widetilde{\mathbf{x}}_{\kappa_{0}}\left(\mathbf{r}_{0}\right)\right\rangle_{D}=\exp \left[-i t_{0} \sqrt{j} \omega_{\kappa_{0}}\left(\mathbf{r}_{0}\right)\right]\left|\mathbf{x}_{\kappa_{0}}\left(\mathbf{r}_{0}\right)\right\rangle_{D}, \widetilde{\mathbf{S}}=$ $e^{-i \pi \widehat{J}_{z} h_{\kappa_{0}} / 2} \mathbf{S},-\widetilde{\mathbf{S}} \equiv e^{-i \pi \widehat{J}_{z}} \widetilde{\mathbf{S}}$, and $\left|0^{\prime}\right\rangle_{D}$ denotes the ground state of the rest of the QD system.

Thus, the completely entangled state has the form of superpositions of NOON states of the extended photon-QD system. It can be obtained using the standard procedure developed for graph states $[68,69]$ with the only difference being that for photons one has to use the spin coherent state $\psi(\mathbf{S})$ instead of qubit states $| \pm\rangle$. The generalization to situations when there are several sets $(i, \kappa, k)$ satisfying $T_{\kappa}^{(k)}\left(\mathbf{r}_{i}\right)=t_{0}$, therefore, is straightforward.

It should be emphasized that the complete entanglement is solely due to the interaction of photons with the $\kappa_{0}$ transition of the QD at $\mathbf{r}_{0}$. The interaction with the other QDs cannot reduce the entanglement but can significantly complicates the structure of the state. In order to see how the "extended" NOON states are present in the state of the whole system, we expand $\left|0^{\prime}\right\rangle_{D}$ over the products of the eigenstates of $\delta_{\kappa}^{x}(\mathbf{r})$. Each product has the form of a sequence of qubits, which correspond to $\left| \pm \mathbf{x}_{\kappa}(\mathbf{r})\right\rangle$. We denote such sequence by $|Q\rangle_{D}$ and rewrite (76) as

$$
\begin{aligned}
\left|\Psi_{0}\right\rangle=\frac{1}{2^{M}} \sum_{Q} & {\left[|\psi(\widetilde{\mathbf{S}}(Q))\rangle\left|\widetilde{\mathbf{x}}_{\kappa_{0}}\left(\mathbf{r}_{0}\right)\right\rangle_{D}\right.} \\
& \left.+|\psi(-\widetilde{\mathbf{S}}(Q))\rangle\left|-\widetilde{\mathbf{x}}_{\kappa_{0}}\left(\mathbf{r}_{0}\right)\right\rangle_{D}\right]|\widetilde{Q}\rangle_{D^{\prime}}
\end{aligned}
$$

where $|\widetilde{Q}\rangle_{D}=\exp \left[-i t_{0} \sum_{\kappa, i}^{\prime} \omega_{\kappa}\left(\mathbf{r}_{i}\right) \sqrt{j} s_{\kappa}\left(\mathbf{r}_{i} \mid Q\right)\right]|Q\rangle_{D}$, with $s_{\kappa}\left(\mathbf{r}_{i} \mid Q\right)=1$ or -1 depending on whether $\left\langle\left.\mathbf{x}_{\kappa}\left(\mathbf{r}_{i}\right)\right|_{D} \mid Q\right\rangle_{D} \neq 0$ or $\left\langle-\left.\mathbf{x}_{\kappa}\left(\mathbf{r}_{i}\right)\right|_{D} \mid Q\right\rangle_{D} \neq 0$ and $|\psi(\widetilde{\mathbf{S}}(Q))\rangle=$ $\exp \left[-i t_{0} \widehat{J}_{z} \sum_{\kappa, i}^{\prime} h_{\kappa} \omega_{\kappa}^{2}\left(\mathbf{r}_{i}\right) s_{\kappa}\left(\mathbf{r}_{i} \mid Q\right) / 2 \sqrt{j}\right]|\psi(\mathbf{S}(Q))\rangle$. The sum signs are primed in order to indicate that the pair $\left(\kappa_{0}, \mathbf{r}_{0}\right)$ is excluded from the summation. 
This consideration shows that completely entangled states are formed due to the interaction with individual QDs and this is why $T_{\kappa}^{(k)}\left(\mathbf{r}_{i}\right)$ do not depend on the total number of QDs. At the same time, similar to what we have seen in the previous section, during the initial stage, different channels of formation of entanglement cooperate and the initial rate of loss of coherence increases with the number of QDs. Indeed, considering the limit $t \sim 0$ we find

$$
J(t)=\exp \left[-\frac{t^{2}}{8 j} \sum_{\kappa, i} \omega_{\kappa}^{2}\left(\mathbf{r}_{i}\right)\right]
$$

This analysis can be straightforwardly generalized to include the case when QDs (all or part of them) are not in resonance with the cavity. In (75), the terms with $\Delta_{\kappa}\left(\mathbf{r}_{i}\right) \neq 0$ does not vanish. In other words the interaction with QDs, which are not in resonance with the cavity modes, do not lead to complete entanglement. Moreover, (78) in this case takes the form

$$
J(t)=\exp \left\{-\frac{t^{2}}{8 j} \sum_{\kappa, i} \omega_{\kappa}^{2}\left(\mathbf{r}_{i}\right)\left[1-\frac{\Delta_{\kappa}^{2}\left(\mathbf{r}_{i}\right)}{\widetilde{B}_{\kappa}^{2}\left(\mathbf{r}_{i}\right)}\right]\right\} .
$$

Thus, the effect of the interaction with off-resonant QDs on initial formation of entanglement is reduced and in the limit of large detuning their contribution into the rate of loss of coherence vanishes $\propto 4 j \omega_{\kappa}^{2}\left(\mathbf{r}_{i}\right) / \Delta_{\kappa}^{2}\left(\mathbf{r}_{i}\right)$. Of course, this result is valid only for relatively short time scales when the effect of neglected terms remains small, as has been discussed above.

\section{Conclusion}

We have considered the basic dynamics of entanglement in the system of coupled second quantized fields in the general context of the problem of solid based sources of entangled light. This compels us to treat entanglement as a property of particles, which are excitations of respective fields, rather than a property of states of fields themselves. The properties of particles and fields are described by different quantities: many-particle density matrix and fields density matrix. We show that while these quantities describe the same state of the physical system they yield different entanglement. This reflects the fact that entanglement depends on the notion of locality: what is the part of the system and what is the complement? These notions are clearly different whether we address properties of particles or fields, in other words, whether we consider the system within the particle or field picture. We show that the same state may be completely entangled in one picture and disentangled in another. States disentangled in the particle picture are superpositions of spin coherent states corresponding to the same one-particle state. Out of these states, only Glauber's coherent states are disentangled in field picture. Taking the inequivalence of two pictures into account, we paid the most attention to the dynamics of entanglement in the particle picture, which is the most relevant for the problem of solid based sources of entangled light.

The first question, which has to be answered, is how is it possible to produce an entangled state of a quantum field?
The circumstance, which motivates this question, is that by a classical source, entangled states (in either picture) cannot be reached out of vacuum. Moreover, we show that in a system of linearly coupled quantized fields, total entanglement (in particle picture) conserves and its dynamics reduces to mere transfer between subsystems. Therefore, in order for initially disentangled states to get entangled it is necessary to have an interaction, a nonlinear dependence of energy on the number of particles. We provide a detailed analysis of the effect of interaction which preserves the population of individual oneparticle states. Such systems admit an exact solution for an arbitrary dimension $n$ of one-particle Hilbert space, while demonstrate nontrivial dynamics of entanglement.

We found that in the simplest $n=2$ case, a generic potential admits an evolution that drives an initially disentangled state through a completely entangled one. When $n>2$, however, only potentials of special form admit such dynamics.

If initially the system is in spin coherent state, the time dependence of entanglement has a characteristic form of a periodic train of Gaussian resonances with the characteristic time of decay of coherence (entanglement time) $\tau \propto$ $1 / \sqrt{N\left(n^{2}-1\right)}$, where $N$ is the total number of particles. By increasing $n$, the time dependence demonstrates a richer structure with an emerging self-similar pattern due to the existence of instances of partial recovery of coherence. Maximal entanglement is shown to be reached at states, which are superpositions of spin coherent states. Completely entangled states correspond to the situation when superimposing states are orthogonal to each other and, thus, belong to the class of NOON states.

If the initial state is disentangled in both particle and field pictures, that is, its Glauber's coherent state, complete entanglement cannot be achieved (if dynamics preserves populations of individual one-particle states). The time dependence of entanglement, however, becomes more complex in this case due to the presence of two entangling channels. One is related to formation of NOON states within each sector with a definite total number of particles and another is due to the dephasing of dynamics within different sectors. Initially, the effects of two channels combine, leading to an increased rate of initial decay of coherence. At long time scales, however, their interplay leads to more complex time dependence of entanglement. For example, if characteristic time scales related to different channels are incommensurate, the loss of initial coherence is irreversible.

Finally, we have considered the evolution of entanglement of photons in a cavity with $M$ two-level quantum dots (QD) in the limit of a large number of photons, $N \gg M$. We show that in relatively short time scale, completely entangled states result from the interaction of photons with QDs tuned in the resonance with the cavity mode. The time when the completely entangled state is reached due to absorption and reemission of a $\kappa$-polarized photon by the $\mathrm{QD}$ at point $\mathbf{r}$ is $T_{\kappa}(\mathbf{r}) \propto \sqrt{N} / \omega_{\kappa}(\mathbf{r})$, where $\omega_{\kappa}(\mathbf{r})$ is the respective Rabi frequency. The interesting feature of these states is that they are a straightforward generalization of the graph states. Since 
they are formed through the interaction with individual QDs, $T_{\kappa}(\mathbf{r})$ do not depend on the number of QDs. However, in the short time limit, $t \ll T_{\kappa}(\mathbf{r})$, different channels cooperate leading to an increasing rate of the loss of coherence with increasing $M$. Detuning a QD away from the resonance leads to its exclusion from the formation of maximally entangled states and reduces its contribution into the initial rate of the loss of coherence.

\section{Conflict of Interests}

The authors declare that there is no conflict of interests regarding the publication of this paper.

\section{Acknowledgments}

The authors acknowledge support from NSF Grant no. ECCS0725514, DARPA/MTO Grant no. HR0011-08-1-0059, NSF Grant no. ECCS-0901784, AFOSR Grant no. FA9550-09-10450, and NSF Grant no. ECCS-1128597.

\section{References}

[1] R. Horodecki, P. Horodecki, and M. H. K. Horodecki, "Quantum entanglement," Reviews of Modern Physics, vol. 81, no. 2, pp. 865-942, 2009.

[2] D. Bouwmeester, A. K. Ekert, and A. Zeilinger, The Physics of Quantum Information, Springer, Berlin, Germany, 2000.

[3] L.-A. Wu, M. S. Sarandy, and D. A. Lidar, "Quantum phase transitions and bipartite entanglement," Physical Review Letters, vol. 93, no. 25, Article ID 250404, 4 pages, 2004.

[4] Y. H. Shih and C. O. Alley, "New type of Einstein-PodolskyRosen-Bohm experiment using pairs of light quanta produced by optical parametric down conversion," Physical Review Letters, vol. 61, pp. 2921-2924, 1988.

[5] Z. Y. Ou and L. Mandel, "Violation of Bell's inequality and classical probability in a two-photon correlation experiment," Physical Review Letters, vol. 61, no. 1, pp. 50-53, 1988.

[6] Y. Shih, "Entangled biphoton source-property and preparation," Reports on Progress in Physics, vol. 66, no. 6, pp. 10091044, 2003.

[7] L. Mandel and E. Wolf, Optical Coherence and Quantum Optics, Cambridge University Press, New York, NY, USA, 1995.

[8] S. Tanzilli, H. De Riedmatten, W. Tittel et al., "Highly efficient photon-pair source using periodically poled lithium niobate waveguide," Electronics Letters, vol. 37, no. 1, pp. 26-28, 2001.

[9] P. G. Kwiat, "New high-intensity source of polarization-entangled photon pairs," Physical Review Letters, vol. 75, pp. 43374341, 1995.

[10] K. Edamatsu, G. Oohata, R. Shimizu, and T. Itoh, "Generation of ultraviolet entangled photons in a semiconductor," Nature, vol. 431, no. 7005, pp. 167-170, 2004.

[11] J. B. Altepeter, E. R. Jeffrey, and P. G. Kwiat, "Phase-compensated ultra-bright source of entangled photons," Optics Express, vol. 13, no. 22, pp. 8951-8959, 2005.

[12] R. M. Stevenson, R. J. Young, P. Atkinson et al., "A semiconductor source of triggered entangled photon pairs," Nature, vol. 439, pp. 179-182, 2006.

[13] R. J. Young, R. M. Stevenson, P. Atkinson, K. Cooper, D. A. Ritchie, and A. J. Shields, "Improved fidelity of triggered entangled photons from single quantum dots," New Journal of Physics, vol. 8, article 29, 2006.

[14] N. Akopian, N. H. Lindner, E. Poem, Y. Berlatzky, J. Avron, and D. Gershoni, "Entangled photon pairs from semiconductor quantum dots," Physical Review Letters, vol. 96, Article ID 130501, 2006.

[15] A. J. Shields, "Semiconductor quantum light sources," Nature Photonics, vol. 1, no. 4, pp. 215-223, 2007.

[16] A. Hayat, P. Ginzburg, and M. Orenstein, "High-rate entanglement source via two-photon emission from semiconductor quantum wells," Physical Review B, vol. 76, Article ID 035339, 2007.

[17] G. Oohata, R. Shimizu, and K. Edamatsu, "Photon polarization entanglement induced by biexciton: experimental evidence for violation of Bell's inequality," Physical Review Letters, vol. 98, Article ID 140503, 4 pages, 2007.

[18] A. Hayat, P. Ginzburg, and M. Orenstein, "Observation of twophoton emission from semiconductors," Nature Photonics, vol. 2, no. 4, pp. 238-241, 2008.

[19] M. Erementchouk and M. N. Leuenberger, "Entanglement of photons due to nonlinear optical response of quantum wells," Physical Review B, vol. 81, no. 19, Article ID 195308, 2010.

[20] V. Vedral, "Quantifying entanglement in macroscopic systems," Nature, vol. 453, no. 7198, pp. 1004-1007, 2008.

[21] M. O. T. Cunha, J. A. Dunningham, and V. Vedral, "Entanglement in single-particle systems," Proceedings of The Royal Society of London A, vol. 463, no. 2085, pp. 2277-2286, 2007.

[22] A. M. Zagoskin, Quantum Theory of Many-Body Systems. Techniques and Applications, Springer, New York, NY, USA, 1998.

[23] R. Paskauskas and L. You, "Quantum correlations in two-boson wave functions," Physical Review A, vol. 64, Article ID 042310, 4 pages, 2001.

[24] X.-G. Wang and B. C. Sanders, "Canonical entanglement for two indistinguishable particles," Journal of Physics A, vol. 38, p. L67, 2005.

[25] N. N. Chung and L. Y. Chew, "Dependence of entanglement dynamics on the global classical dynamical regime," Physical Review E, vol. 80, no. 1, Article ID 016204, 7 pages, 2009.

[26] S. D. Bartlett and H. M. Wiseman, "Entanglement constrained by superselection rules," Physical Review Letters, vol. 91, no. 9, Article ID 097903, 4 pages, 2003.

[27] M. A. Can, A. Klyachko, and A. Shumovsky, "Single-particle entanglement," Journal of Optics B, vol. 7, no. 2, pp. L1-L3, 2005.

[28] S. J. van Enk, "Single-particle entanglement," Physical Review A, vol. 72, Article ID 064306, 3 pages, 2005.

[29] A. Drezet, "Comment on "Single-particle entanglement'”' Physical Review A, vol. 74, Article ID 026301, 2 pages, 2006.

[30] M. Pawłowski and M. Czachor, "Degree of entanglement as a physically ill-posed problemml: the case of entanglement with vacuum," Physical Review A, vol. 73, no. 4, Article ID 042111, 7 pages, 2006.

[31] A. Perelomov, Generalized Coherent States and Their Applications, Springer, Berlin, Germany, 1986.

[32] J.-P. Gazeau, Coherent States in Quantum Physics, Wiley-VCH, Weinheim, Germany, 2009.

[33] W. M. Zhang, D. H. Feng, and R. Gilmore, "Coherent states: theory and some applications," Reviews of Modern Physics, vol. 62, no. 4, pp. 867-927, 1990.

[34] A. N. Boto, P. Kok, D. S. Abrams, S. L. Braunstein, C. P. Williams, and J. P. Dowling, "Quantum interferometric optical 
lithography: exploiting entanglement to beat the diffraction limit," Physical Review Letters, vol. 85, pp. 2733-2736, 2000.

[35] X. Wang, B. C. Sanders, and S.-H. Pan, "Entangled coherent states for systems with $S U(2)$ and $S U(1,1)$ symmetries," Journal of Physics A, vol. 33, no. 41, pp. 7451-7467, 2000.

[36] B. C. Sanders, "Review of entangled coherent states," Journal of Physics A, vol. 45, no. 24, Article ID 244002, 22 pages, 2012.

[37] H. Makela and K.-A. Suominen, "Inert States of Spin-S Systems," Physical Review Letters, vol. 99, Article ID 190408, 2007.

[38] D. J. H. Markham, "Entanglement and symmetry in permutation-symmetric states," Physical Review A, vol. 83, Article ID 042332, 2011.

[39] J. J. Sakurai, Modern Quantum Mechanics, Addison-Wesley, Reading, Mass, USA, 1994.

[40] W. K. Wootters, "Entanglement of formation of an arbitrary state of two qubits," Physical Review Letters, vol. 80, pp. 22452248, 1998.

[41] H. Georgi, Lie Algebras in Particle Physics, WestView Press, Boulder, Colo, USA, 1999.

[42] H. Aschauer, J. Calsamiglia, M. Hein, and H. J. Briegel, "Local invariants for multi-partite entangled states allowing for a simple entanglement criterion," Quantum Information \& Computation, vol. 4, no. 5, pp. 383-395, 2004.

[43] A. A. Klyachko, B. Oztop, and A. S. Shumovsky, "Quantification of entanglement via uncertainties," Physical Review A, vol. 75, Article ID 032315, 6 pages, 2007.

[44] A. Klyachko, "Dynamical symmetry approach to entanglement," in Physics and Theoretical Computer Science, vol. 7, pp. 25-54, IOS, Amsterdam, The Netherlands, 2007.

[45] T.-C. Wei, "Maximal entanglement versus entropy for mixed quantum states," Physical Review A, vol. 67, Article ID 022110, 12 pages, 2003.

[46] M. Mathur and I. Raychowdhury, " $S U(N)$ coherent states and irreducible Schwinger bosons," Journal of Physics A, vol. 44, no. 3, Article ID 035203, 14 pages, 2011.

[47] M. Mathur, I. Raychowdhury, and R. Anishetty, "SU(N) irreducible Schwinger bosons," Journal of Mathematical Physics, vol. 51, no. 9, Article ID 093504, 14 pages, 2010.

[48] V. Vedral, "Entanglement in the second quantization formalism," Central European Journal of Physics, vol. 2, pp. 289-306, 2003.

[49] B. He and J. A. Bergou, "Coherent-states engineering with linear optics: possible and impossible tasks," Physical Review A, vol. 77, Article ID 053818, 2008.

[50] B. C. Sanders, "Quantum dynamics of the nonlinear rotator and the effects of continual spin measurement," Physical Review A, vol. 40, pp. 2417-2427, 1989.

[51] K. Tara, G. S. Agarwal, and S. Chaturvedi, "Production of Schrödinger macroscopic quantum-superposition states in a Kerr medium," Physical Review A, vol. 47, no. 6, pp. 5024-5029, 1993.

[52] M. Kitagawa and M. Ueda, "Squeezed spin states," Physical Review A, vol. 47, no. 6, pp. 5138-5143, 1993.

[53] A. Melikidze, V. V. Dobrovitski, H. A. De Raedt, M. I. Katsnelson, and B. N. Harmon, "Parity effects in spin decoherence," Physical Review B, vol. 70, no. 1, Article ID 014435, 2004.

[54] J. Lages, V. V. Dobrovitski, M. I. Katsnelson, H. A. De Raedt, and B. N. Harmon, "Decoherence by a chaotic many-spin bath," Physical Review E, vol. 72, no. 2, Article ID 026225, 2005.

[55] S. Yuan, M. I. Katsnelson, and H. De Raedt, "Origin of the canonical ensemble: thermalization with decoherence," Journal of the Physical Society of Japan, vol. 78, no. 9, Article ID 094003, 2009.

[56] O. Gittsovich, P. Hyllus, and O. Guhne, "Multiparticle covariance matrices and the impossibility of detecting graph-state entanglement with two-particle correlations," Physical Review A, vol. 82, Article ID 032306, 2010.

[57] S. J. van Enk, "Entanglement capabilities in infinite dimensions: multidimensional entangled coherent states," Physical Review Letters, vol. 91, no. 1, Article ID 017902, 4 pages, 2003.

[58] F. Iachello, Lie Algebras and Applications, vol. 708 of Lecture Notes in Physics, Springer, Berlin, Germany, 2006.

[59] S.-B. Zheng, "One-step synthesis of multiatom GreenbergerHorne-Zeilinger states," Physical Review Letters, vol. 87, Article ID 230404, 2001.

[60] A. Klein and E. R. Marshalek, "Boson realizations of Lie algebras with applications to nuclear physics," Reviews of Modern Physics, vol. 63, no. 2, pp. 375-558, 1991.

[61] F. Iachello and R. D. Levine, Algebraic Theory of Molecules, Oxford University Press, New York, NY, USA, 1995.

[62] E. Fradkin, Field Theories of Condensed Matter Systems, vol. 82, Addison-Wesley, Redwood City, Calif, USA, 1991.

[63] B. C. Berndt and R. J. Evans, "The determination of Gauss sums," Bulletin of the American Mathematical Society, vol. 5, no. 2, pp. 107-129, 1981.

[64] B. C. Berndt, R. J. Evans, and K. S. Williams, Gauss and Jacobi Sums, Wiley-Interscience, Toronto, Canada, 1997.

[65] B. W. Shore and P. L. Knight, "The Jaynes-Cummings model," Journal of Modern Optics, vol. 40, no. 7, pp. 1195-1238, 1993.

[66] M. Scully and M. Zubairy, Quantum Optics, Cambridge University Press, Cambridge, UK, 1997.

[67] M. Erementchouk and M. N. Leuenberger, "Complex dynamics of photon entanglement in the two-mode Jaynes-Cummings model," Nanotechnology, vol. 21, no. 27, Article ID 274019, 2010.

[68] H. J. Briegel and R. Raussendorf, "Persistent entanglement in arrays of interacting particles," Physical Review Letters, vol. 86, no. 5, pp. 910-913, 2001.

[69] M. Hein, J. Eisert, and H. J. Briegel, "Multiparty entanglement in graph states," Physical Review A, vol. 69, no. 6, Article ID 062311, 20 pages, 2004. 


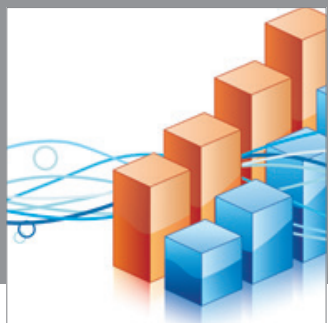

Advances in

Operations Research

mansans

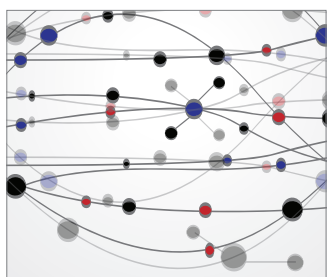

The Scientific World Journal
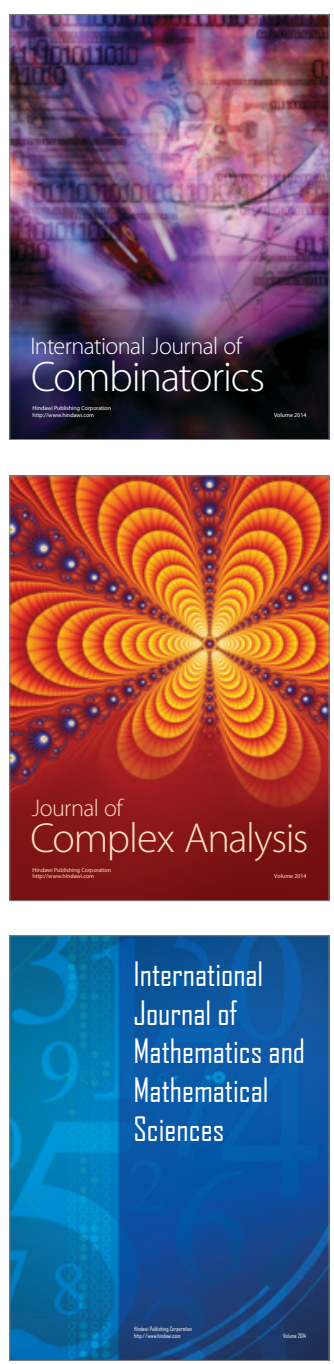
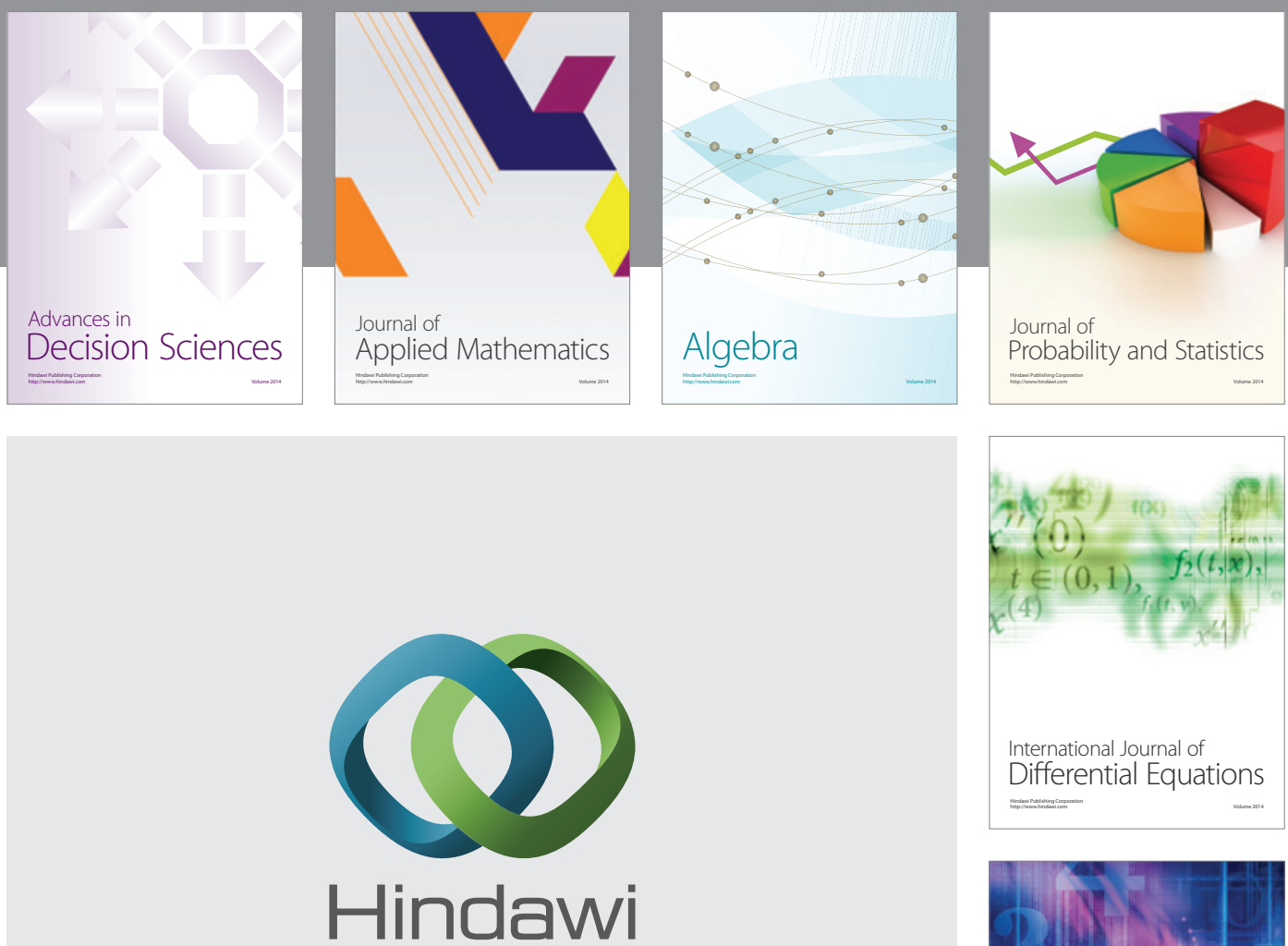

Submit your manuscripts at http://www.hindawi.com
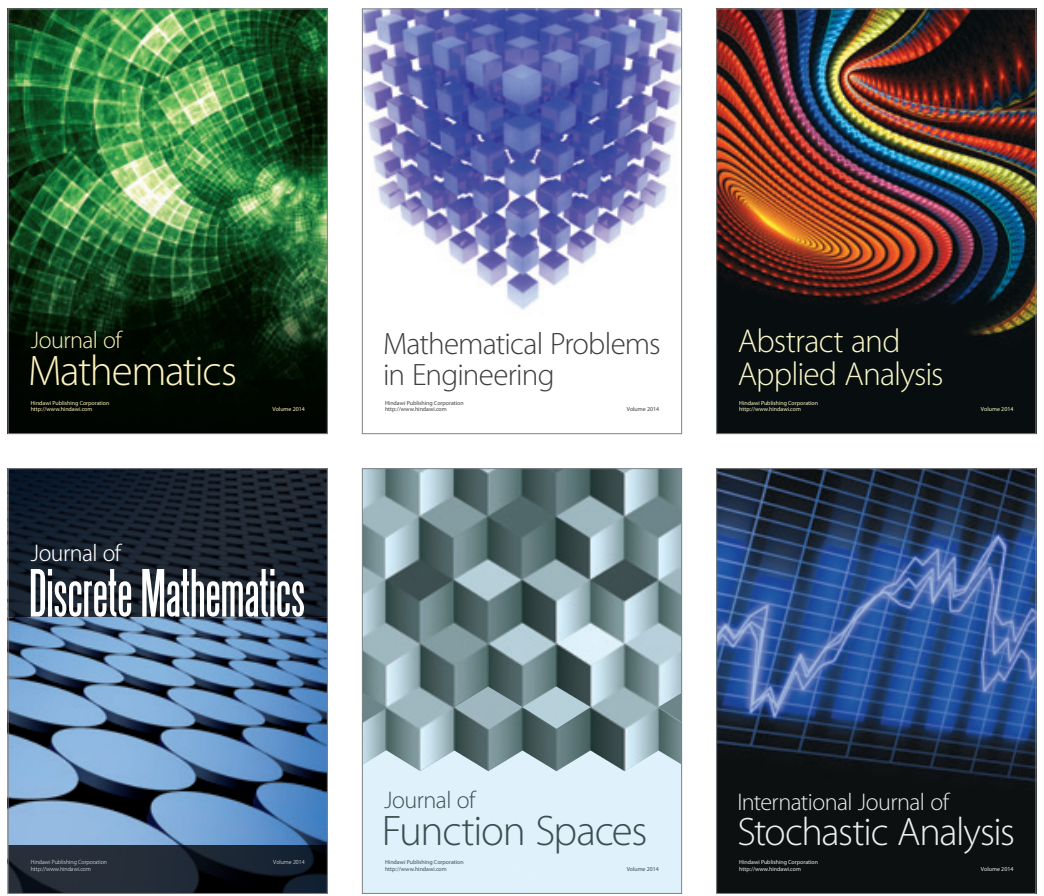

Journal of

Function Spaces

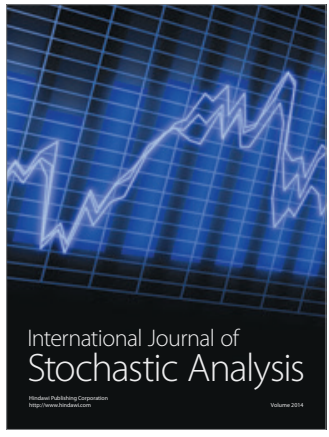

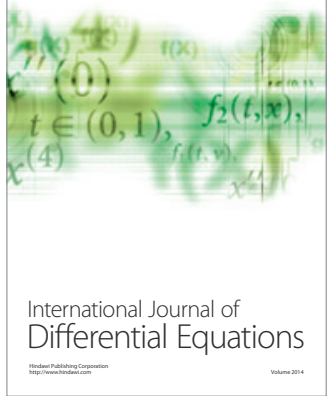
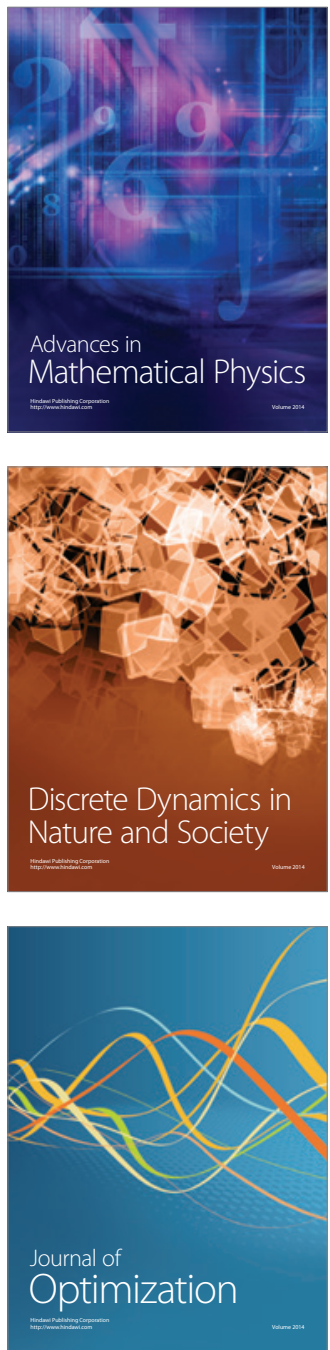